Portland State University

PDXScholar

$1-1-2010$

\title{
Mitochondrial Inheritance and Natural Phenotypic Variation Among Caenorhabditis briggsae Populations
}

Anna Luella Coleman-Hulbert

Portland State University

Follow this and additional works at: https://pdxscholar.library.pdx.edu/open_access_etds

Part of the Biology Commons

Let us know how access to this document benefits you.

\section{Recommended Citation}

Coleman-Hulbert, Anna Luella, "Mitochondrial Inheritance and Natural Phenotypic Variation Among Caenorhabditis briggsae Populations" (2010). Dissertations and Theses. Paper 340.

https://doi.org/10.15760/etd.340

This Thesis is brought to you for free and open access. It has been accepted for inclusion in Dissertations and Theses by an authorized administrator of PDXScholar. Please contact us if we can make this document more accessible: pdxscholar@pdx.edu. 
Mitochondrial Inheritance and Natural Phenotypic Variation among

Caenorhabditis briggsae Populations

by

Anna Luella Coleman-Hulbert

A thesis submitted in partial fulfillment of the requirements for the degree of

Master of Science

in

Biology

Thesis Committee:

Suzanne Estes, Chair

Bradley A. Buckley

Todd Rosenstiel

Portland State University

2010 


\begin{abstract}
Mutations affecting the mitochondrial electron transport chain cause numerous neurodegenerative disorders in humans and affect longevity in other organisms. A natural model system to study the relationship between mitochondrial function and aging within an evolutionary or population genetic context has been lacking. Natural populations of Caenorhabditis briggsae nematodes were recently found to harbor mitochondrial genetic variation with likely functional consequences for aging. Specifically, C. briggsae isolates containing high frequencies of a deletion mutation affecting the mitochondrial $N A D H$ dehydrogenase 5 (ND5) gene were found to have reduced reproductive fitness and lifespan and elevated levels of mutagenic superoxide. Here, rates of growth and aging and aerobic respiratory capacity were evaluated in several isolates spanning the range of mitochondrial genetic variation in this species. There is considerable variation among isolates for all measured traits, although the observed relationships between isolatespecific trait means and ND5 deletion frequency did not always conform to my expectations. In an effort to determine whether the among-isolate phenotypic variation is due to mitochondrial rather than to nuclear genetic variation, inter-population hybrids of C. briggsae were created and compared to the progenitor isolates. Surprisingly, evidence for paternal mitochondrial inheritance was detected in many of these hybrid lines. Where mitochondrial genomes were maternally inherited as expected, intergenomic epistasis appears to contribute to fitness, longevity, and aging in this species.
\end{abstract}




\section{Acknowledgements}

Thanks especially to S. Estes for introducing me to the $C$. briggsae system, and for seemingly endless support. Thanks also to J. B. Knapp and K. A. Hicks for excessive amounts of personal and professional support. 


\section{Table of Contents}

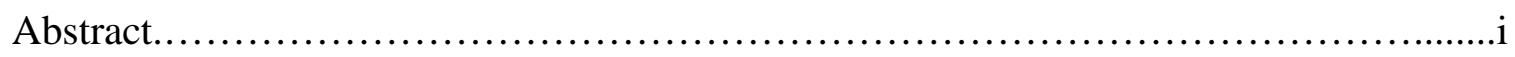

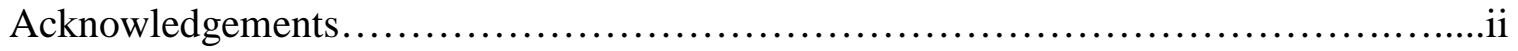

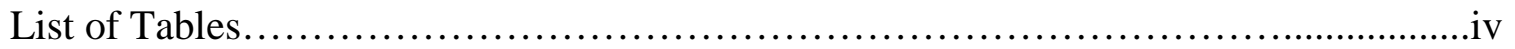

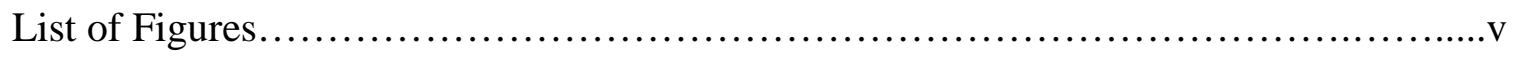

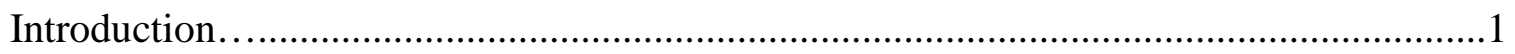

Chapter 1 - Phenotypic Variation among Natural Isolates of Caenorhabditis briggsae...6 Background.......................................................6

Methods.............................................................. 11

Results ............................................................. 16

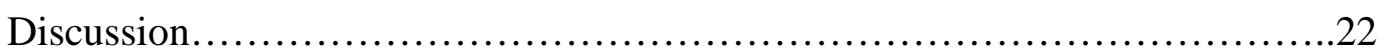

Chapter 2 - Inter-Population Hybrids of Caenorhabditis briggsae Natural Isolates......27

Background.....................................................27

Methods............................................................. 31

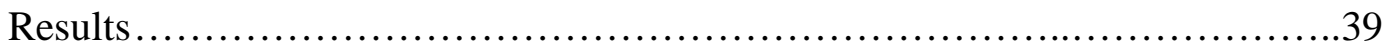

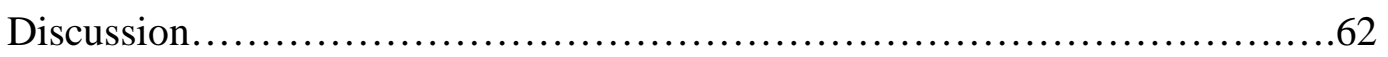

Conclusion................................................................66

References..............................................................68 


\section{List of Tables}

Table 1. Test statistics per phenotype measured among nine natural isolates of Caenorhabditis briggsae.

Table 2. Expected ND5 deletion heteroplasmy levels in C. briggsae hybrids.

Table 3. Test statistics per trait from comparisons between pairs of natural isolate $C$. briggsae progenitors used for hybridization.

Table 4. Test statistics per trait from comparisons between or among replicates of mHK104 x nEG4181 hybridization, and between the replicates pooled and the maternal isolate, $\mathrm{HK} 104$.

Table 5. Test statistics per trait from comparisons between or among replicates of mEG4181 x nHK104 hybridization, and between the replicates pooled and the maternal isolate, EG4181.

Table 6. Test statistics per trait from comparisons between or among replicates of mHK105 x nPB800 hybridization, and between the replicates pooled and the maternal isolate, HK105

Table 7. Test statistics per trait from comparisons between or among replicates of mPB800 x nHK105 hybridization, and between the replicates pooled and the maternal isolate, $\mathrm{PB} 800$.

Table 8. Expectations of heteroplasmy levels and outcomes from crossing C. briggsae natural isolates with high and low ND5 deletion levels. 


\section{List of Figures}

Figure 1. Caenorhabditis briggsae mitochondrial genome...........................................8

Figure 2. Caenorhabditis briggsae intraspecific phylogeny........................................

Figure 3. Pharynx in Caenorhabditis briggsae adult.....................................................12

Figure 4. Caenorhabditis briggsae at fourth instar (L4)............................................13

Figure 5. Adult pharyngeal pumping rates of $C$. briggsae .........................................16

Figure 6. Length of $C$. briggsae larvae at 14, 26, 34, and 42 hours after hatching..........18

Figure 7. Length of $C$. briggsae adults at 0,6 , and 12 days post-L4.............................19

Figure 8. Whole-organism rates of oxygen consumption of $C$. briggsae .......................20

Figure 9. C. briggsae natural isolates selected for hybridization..................................32

Figure 10. Total reproductive output for each parental and introgressed line of natural $C$.

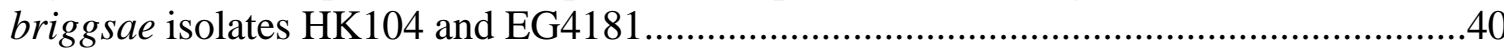

Figure 11. Total reproductive output for each parental and introgressed line of natural $C$.

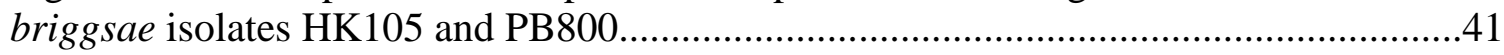

Figure 12. Lifespan in days since hatching for each parental and introgressed line of natural C. briggsae isolates HK104 and EG4181.......................................................42

Figure 13. Lifespan in days since hatching for each parental and introgressed line of natural $C$. briggsae isolates HK105 and PB800...........................................................43

Figure 14. Adult pharyngeal pumping for each parental and two introgressed line of each reciprocal cross of natural C. briggsae isolates HK104 and EG4181.............................44

Figure 15. Adult pharyngeal pumping for each parental and two introgressed line of each reciprocal cross of natural $C$. briggsae isolates HK105 and PB800..............................45

Figure 16. Length of larvae from each parental and introgressed line of natural $C$. briggsae isolates HK104 and EG4181. 
Figure 17. Length of larvae from each parental and introgressed line of natural $C$.

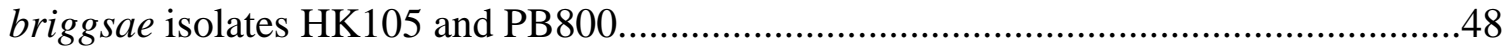

Figure 18. Length of adults from each parental and introgressed line of natural $C$. briggsae isolates HK104 and EG4181 .......................................................................50

Figure 19. Length of adults from each parental and introgressed line of natural $C$.

briggsae isolates HK105 and PB800...........................................................................51

Figure 20. Whole-organism oxygen consumption of young adults for each parental and introgressed line of natural C. briggsae isolates HK104 and EG4181 ..............................52

Figure 21. Whole-organism oxygen consumption of young adults for each parental and introgressed line of natural $C$. briggsae isolates HK105 and PB800................................53 


\section{INTRODUCTION}

Mutations affecting mitochondrial DNA (mtDNA) and function are associated with a variety of metabolic and aging-related diseases across taxa (Chinnery and Turnbull, 2000; DiMauro and Schon, 2003; Smeitink et al., 2003; Taylor and Turnbull, 2005; Wallace, 2005). Nuclear and mitochondrial gene products interact to form the energy-producing mitochondrial electron transport chain (ETC), and mutations affecting these gene functions tend to present as various neurodegenerative disorders in humans (Ozawa, 1995; Pitkanen and Robinson, 1996; DiMauro and Schon, 2003; Smeitink et al., 2003; Taylor and Turnbull, 2005). In general, mtDNA mutates faster than nuclear DNA (nDNA) due to: 1) increased replication compared to nDNA over the lifetime of an individual, 2) lack of repair enzymes, and 3) proximity to reactive oxygen species (ROS) that are natural by-products of aerobic metabolism in mitochondria (Lightowlers et al., 1997; Ballard and Whitlock, 2004; Baer et al., 2007). When mutated, mitochondrial genes tend to exist in a heteroplasmic state; that is, wild type mitochondrial genomes exist alongside mutant genomes within the same organism, cell, or organelle (Chinnery, 2000; Smeitink et al., 2003; Lemire, 2005). Several defects associated with mtDNA mutations demonstrate a threshold effect of heteroplasmy whereby a certain fraction of mutated organellar genomes must be present for deleterious phenotypes to manifest (Rossignol et al., 2003; Smeitink et al., 2003; Ventura et al., 2006). 
Introduction

MtDNA mutations can be inherited, or arise spontaneously within somatic tissues and accumulate over the lifetime of an individual (Solignac et al., 1987; Chinnery, 2000; Tsang and Lemire, 2002; Smeitink et al., 2003; Liau et al., 2007), and increases in mtDNA mutations and heteroplasmy are known to occur with age in many cases (CorralDebrinski et al., 1992.; Ozawa, 1995; Melov et al., 1997; Chomyn and Attardi, 2003; Krishnan et al., 2008). The precise mechanism for such increases in mutation heteroplasmy is not known, although it has been suggested that, when deletions are present within a mitochondrial genome, such a molecule could exhibit a replicative advantage over the intact (wild type) genome due simply to the shorter time required to replicate a shorter deletion-bearing sequence (Yoneda et al., 1992; Melov et al., 1995; Moraes, 2001; Diaz et al., 2002.; however, see Solignac et al., 1987). In addition to this age-related prevalence of mtDNA mutations, mutations of mtDNA and mitochondrial function have been implicated as causal agents in the aging process (Bandy and Davison, 1990; Ozawa, 1995; Lee and Wei, 2007; Alexeyev et al., 2004; Chomyn and Attardi, 2003; Ventura et al., 2006; Sedensky and Morgan, 2006a; Sedensky and Morgan, 2006b). Mutations leading to the dysfunction of mitochondria, specifically of the ETC, and changes in ROS production can affect rates of aging and longevity across organisms (Ozawa, 1995; Chomyn and Attardi, 2003; Tsang and Lemire, 2003; Ventura et al., 2006; Sedensky and Morgan, 2006a; Sedensky and Morgan, 2006b; Hiona and Leeuwenburgh, 2008). 
Introduction

The long-standing free radical theory for aging purports that the degenerative features of senescence are largely due to the accumulation of lipids, proteins, and DNA that have been damaged by endogenously-produced ROS (Harman, 1956; Beckman and Ames, 1998; Alexeyev et al., 2004; Balaban et al., 2005). Mitochondria have been deemed the primary source of endogenous ROS (Murphy, 2009), which has placed these organelles in the spotlight of much theoretical and empirical aging research. Some predict that a vicious cycle may occur within mitochondria where natural ROS production generates mtDNA damage, this damage leads to subsequent dysfunction of the organelle thus increasing ROS production, and so on (Linnane et al., 1989; Bandy and Davison, 1990; Alexeyev et al., 2004; although, see de Grey, 2005, and Hiona and Leeuwenburgh, 2008). To understand the interplay of mitochondrial function, ROS production, aging, and longevity, research has utilized in vitro, in vivo, and in situ methods on models as diverse as snakes and 'cybrids' (cell cultures with replaced mtDNA populations) (Ozawa, 1995; Pitkanen and Robinson, 1996; Luo et al., 1997; Melov et al., 1997; Inoue et al., 2000; Robert et al., 2007). However, the fact remains that we have a limited understanding of the evolutionary forces underlying the aging process and mitochondrial genetics.

Ease of laboratory maintenance and manipulation, and our thorough understanding of Caenorhabditis elegans genetics have made this nematode a powerful model organism for studies of aging and many other biological processes. Several single C. elegans genes involved in mitochondrial function and biogenesis (most of which have 
Introduction

human orthologues) have been identified through chemical mutagenesis screens as affecting metabolism and longevity. However, a major hurdle to understanding the link between mitochondrial mtDNA damage, mitochondrial dysfunction, and the aging process has been the lack of animal model systems with relevant naturally occurring mitochondrial genetic variation. Howe and Denver (2008) recently characterized a mitochondrial deletion mutation that segregates among natural populations of the soil nematode Caenorhabditis briggsae, a sister species to $C$. elegans for which most of the same molecular techniques and genetic tools are available. This mutation deletes over 700bp of the ETC complex I subunit NADH dehydrogenase 5 (ND5). Furthermore, the deletion-bearing mtDNA genomes comprise between 0 to over $50 \%$ of the total mitochondrial genome population depending upon the $C$. briggsae natural isolate. The natural variation present among isolates of $C$. briggsae for a clinically relevant mtDNA deletion provides an excellent opportunity to characterize aging features that are correlated to a naturally occurring and presumably deleterious mtDNA mutation.

Findings from other complex I mutants indicate that the deletion affecting ND5 in C. briggsae can be expected to increase ROS production and decrease ETC efficacy, and thereby impact whole organismal function (Pitkanen and Robinson, 1996; Luo et al., 1997; Bai et al., 2000; Kayser et al., 2004; Janssen et al., 2006; Park et al., 2009). Accordingly, heteroplasmy in C. briggsae is negatively correlated with reproductive output (Howe and Denver, 2008), population growth rate, and lifespan (Estes et al., in review). Additionally, in vivo ROS production exhibits a non-linear relationship to $C$. 
Introduction

briggsae heteroplasmy; however, ATP content exhibits no relationship with ND5 deletion level (Estes et al., in review). Under the threshold effect and free radical theories, once $C$. briggsae ND5 deletion heteroplasmy reaches a certain level, physiological function should decline and rates of aging should increase due to increased ROS production. Toward the goal of testing these ideas, I have measured various physiological and aging parameters in C. briggsae natural isolates to evaluate the deleterious impact of the ND5 deletion. These data are reported in Chapter 1 of this thesis. Next, to establish that observed variation for traits measured among natural C. briggsae isolates is not due to among-isolate nuclear genetic variation, multiple replicates of reciprocal 'mitochondrial replacement' lines were created by crossing C. briggsae from high- and lowheteroplasmy isolates. All traits were measured in hybrids as for natural parental isolates assuming that mitochondria are inherited maternally and mtDNA variation explains the observed phenotypic differences between natural $C$. briggsae isolates. These experiments are the subject of Chapter 2 of this thesis. Overall, my findings suggest that partial deletion of ND5 may significantly impact life history and aging in C. briggsae, but not certain components of aerobic metabolic function, and that paternal inheritance of mitochondria and cytonuclear conflict can occur in hybrids of C. briggsae natural populations. 


\section{CHAPTER 1}

Phenotypic Variation among Natural Isolates of Caenorhabditis briggsae

\section{BACKGROUND}

Mitochondria are essential for cellular energy metabolism in eukaryotes, and mutations affecting mitochondrial function have been associated with numerous human neurodegenerative disorders (Ozawa, 1995; Pitkanen and Robinson, 1996; Chinnery and Turnbull, 2000; DiMauro and Schon, 2003; Smeitink et al., 2003; Taylor and Turnbull, 2005; Wallace, 2005; Sedensky and Morgan, 2006; Hiona and Leeuwenburgh, 2008). Across taxa, mitochondrial mutations are associated with altered metabolic activity, rates of aging, and longevity (Ozawa, 1995; Pitkanen and Robinson, 1996; Luo et al., 1997; Melov et al., 1997; Inoue et al., 2000; Robert et al., 2007). Since mitochondria are the primary endogenous source of reactive oxygen species (ROS) (Murphy, 2009), these small but plentiful organelles have been a key target for studies aimed at understanding aging, a ubiquitous process thought to be linked to increases in cellular ROS and mitochondrial mutations (Miwa et al., 2008).

Our extensive knowledge of Caenorhabditis elegans biology and the genetic and genomic tools available for this nematode make it an ideal organism for understanding the causative role of mtDNA mutations and mitochondrial function in aging and 
longevity (Tsang and Lemire, 2003; Sedensky and Morgan, 2006a; Sedensky and Morgan, 2006b; Ventura et al., 2006). There are four main genetic pathways known to affect longevity in C. elegans: insulin-like signaling, dietary restriction, inhibition of protein translation, and a mitochondrial pathway (reviewed in Houthoofd and Vanfleteren, 2007). A recent RNAi screen for genes affecting longevity in C. elegans found that about $25 \%$ of new candidate genes (annotated with a possible function) were involved in metabolism, many with orthologues in flies, mice, and humans, and that about half of these genes correspond to subunits of the electron transport chain (ETC) (Hamilton et al., 2005). In particular, RNAi leading to impaired mitochondria and increased longevity also correlate with decreased oxygen consumption and ATP content in C. elegans (Lee et al., 2003). Progeria-like phenotypes and decreased lifespan have been observed in C. elegans with knockdown of mitochondrial heat shock protein 70, an import motor essential to energy generation and mitochondrial biogenesis in eukaryotic cells (Kimura et al., 2007). Inhibition of oxidative phosphorylation at various ETC complexes with RNAi prior to adulthood increases C. elegans longevity (Dillin et al., 2002; Rea et al., 2007). In general, C. elegans mitochondria-associated gerontogenes affect ROS production and mitochondrial oxidative phosphorylation in addition to lifespan (Kayser et al., 2004; Sedensky and Morgan, 2006a; Sedensky and Morgan, 2006b). Although a great degree of homology exists between ETC genes of humans and C. elegans (see Table 1 in Tsang and Lemire, 2003), a natural model is lacking for studying the relationship between mitochondrial mutations, ROS, and aging. 


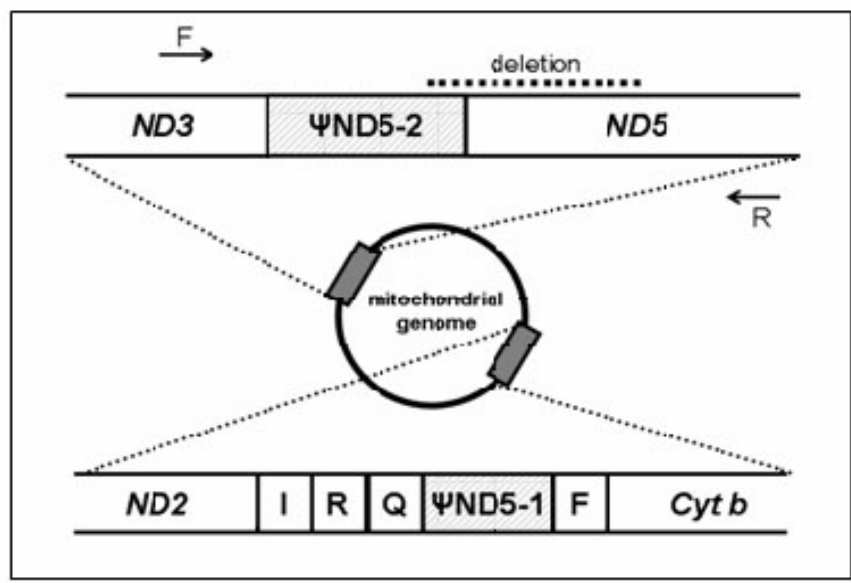

Caenorhabditis briggsae nematodes isolated from geographically distinct

populations around the world have been found to harbor a naturally-occurring heteroplasmic mtDNA deletion (Howe and Denver, 2008; Fig. 1). When present, the deletion removes nearly half of the NADH-dehydrogenase 5 (ND5) gene and over 200 amino acids from the protein product, many of which are highly conserved in C. elegans, Drosophila, and humans. The $C$. briggsae ND5 deletion is thought to occur during genomic replication due to direct repeat sequences that flank the deleted region located within ND5 and within the upstream $\Psi$ ND5-2 non-coding element ( $c f$. Lunt and Hyman, 1997). In C. briggsae isolated from Kenya that lack $\Psi$ ND5-2, no genomes harbor the partial deletion of ND5, whereas isolates that do harbor the non-coding pseudogene have ND5 deletion heteroplasmy levels ranging from 0 to $50 \%$ (Fig. 2). Putative compensatory mutations within $\Psi$ ND5-2 are found in some isolates of the temperate clade and are thought to decrease the likelihood of the deletion event (Howe and Denver, 2008). Based on studies of other complex I mutant genotypes, including other mutations within ND5 
(Pitkanen and Robinson, 1996; Luo et al., 1997; Bai et al., 2000; Kayser et al., 2004;

Janssen et al., 2006; Park et al., 2009), the heteroplasmic deletion in C. briggsae is presumed to decrease complex I efficiency and increase ROS production. Although the truncated ND5 gene is transcribed in C. briggsae, its structure, expression pattern, and degree of functionality have not been determined.

Previous studies found a negative correlation between fecundity and ND5 deletion heteroplasmy level among $C$. briggsae isolates suggesting that the ND5 deletion negatively impacts fitness in C. briggsae (Howe and Denver, 2008). Estes et al. (in review) found this same correlation and, furthermore, that $C$. briggsae heteroplasmy is negatively correlated to population growth rates and lifespan. Additionally, C. briggsae ATP content, in vitro ROS production, and exogenous oxidative stress resistance vary significantly with heteroplasmy; however, only the latter was significantly correlated to heteroplasmy in a negative fashion (Estes et al., in review). Use of fluorogenic dyes

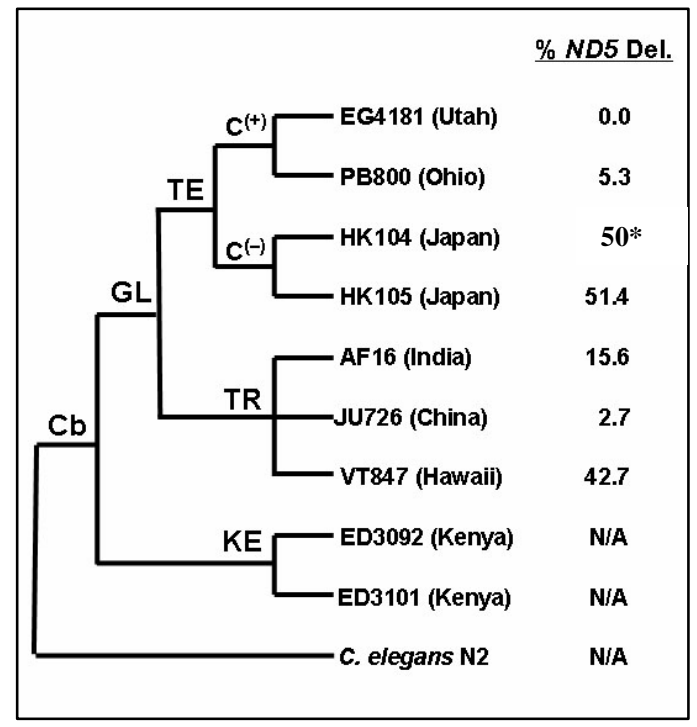

Figure 2. Caenorhabditis briggsae intraspecific phylogeny. $\mathrm{GL}=$ global intraspecific superclade; $\mathrm{KE}=$ Kenya clade; TE and TR $=$ temperate and tropical subclades of GL. $\mathrm{C}(+)=$ temperate-clade isolates bearing compensatory $\Psi$ ND5-2 alleles; $\mathrm{C}(-)=$ those bearing ancestral alleles. \% ND5 Del. indicates isolatespecific percentages of total mitochondrial genomes that harbor ND5 deletions, as determined by qPCR (Howe and Denver, 2008). * The HK104 strain used here was the inbred progenitor of mutationaccumulation lines (Baer et al., 2005) found to harbor high ND5 deletion levels similar to those of HK105 (Dee Denver, pers. comm.). 
revealed a non-linear relationship between whole-worm superoxide production and $C$. briggsae isolate-specific heteroplasmy; i.e. high- and non-heteroplasmic isolates contain more ROS than midlevel deletion isolates (Estes et al., in review). Use of alternative energy-harvesting pathways or variation in oxidant scavenging among isolates may explain the lack of correlation between $C$. briggsae heteroplasmy and both ATP content and in vitro hydrogen peroxide production ( $c f$. Rea, 2005).

To establish $C$. briggsae as a natural model system for examining interactions between aging, heteroplasmy and ETC function, several metabolic and aging-related phenotypes have been examined in nine natural isolates spanning the range of ND5 deletion heteroplasmy in C. briggsae (Fig. 2). Specifically, declines in pharyngeal pumping rate (an indication of muscle deterioration) have been examined to elucidate whether the ND5 deletion affects the aging process in C. briggsae. Additionally, rates of oxygen consumption have been quantified to determine if the ND5 deletion affects aerobic respiratory capacity, and larval and adult growth rates have been examined to determine whether the ND5 deletion in C. briggsae retards cell cycle progression and therefore development. Based on previous studies of other ETC mutations in C. elegans (Dillin et al., 2002; Lee et al., 2003; Kayser et al., 2004), ND5-deletion heteroplasmy in C. briggsae is expected to correlate positively to aging and negatively to metabolism. 


\section{METHODS}

Nematode Strains and Maintenance

To examine the impact on aging and total physiology of a naturally-occurring partial deletion of the mtDNA-encoded ND5 protein coding gene in C. briggsae, nine natural isolates from geographically diverse populations were selected for study in order to capture the full range of variation in ND5 deletion heteroplasmy level ( 0 to $\sim 50 \%$ ) (Fig. 2; strains were provided by D. R. Denver, OSU). Unless otherwise noted, nematodes were grown under standard laboratory conditions at $25^{\circ} \mathrm{C}$ on $15 \mathrm{~mm} \mathrm{NGM}$ plates seeded with Escherichia coli strain HB101 as a food source.

\section{Pharyngeal pumping}

Caenorhabditid nematodes ingest bacteria using rhythmic contractions of the pharynx (Fig. 3) - a neuromuscular organ comprising 20 muscle cells and 20 neurons that is easily viewed using light microscopy (Albertson and Thomson, 1976). As in humans, muscle deterioration occurs with age in nematodes, and sarcopenia of the pharynx has been associated with aging in C. elegans (Garigan et al., 2002; Herndon et al., 2002). Additionally, the decline of pharyngeal pumping is a strong biomarker for chronological age in C. elegans (Bolanowski et al., 1981; Huang et al., 2004). Therefore, the decline of pharyngeal pumping rates in $C$. briggsae over adulthood have been measured here as a proxy for aging. 


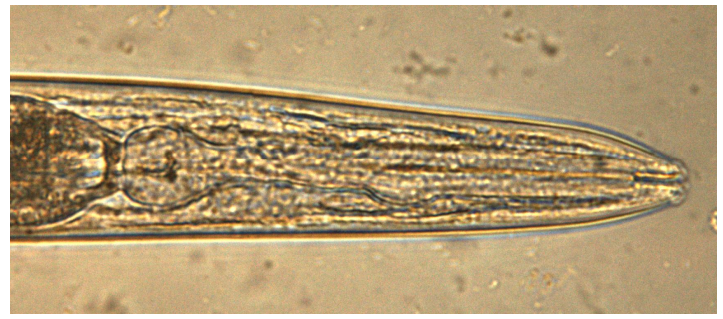

Figure 3. Pharynx in Caenorhabditis briggsae adult.

Pharyngeal pumping assays were performed at room temperature (about $22^{\circ} \mathrm{C}$ ) on $15 \mathrm{~mm}$ NGM plates. Pharyngeal pumping rates were determined for the same 10 to 20 hermaphrodites per isolate every other day of adulthood starting two days past the fourth larval stage (L4 + 2 days; L4 stage confirmed visually by crescent at vulva) until death. Rates were determined by terminal bulb pumps per three 5-second intervals and converted to pumps per minute. Decline in pharyngeal pumping rates were calculated between days 2 and 12 and between days 2 and 20. During the reproductive period, individuals were removed from their offspring after pharyngeal pumping measures.

\section{Growth}

If the ND5 deletion negatively affects ETC functioning in C. briggsae, individuals from isolates harboring high $N D 5$ deletion levels are expected to exhibit slower rates of growth and to attain smaller maximum body sizes than low or zero-deletion isolates ( $c f$. Dillon et al., 2002, and Rea et al., 2007). To test this, different aspects of body size and growth rates were quantified for replicate animals from each natural isolate.

For logistical reasons, growth rates were characterized for larvae and adults separately. For larval measurements, populations were age synchronized by allowing 10- 
20 gravid hermaphrodites per isolate to lay eggs on fresh $15 \mathrm{~mm}$ NGM plates with $E$. coli OP50-1 for two hours. From these age-synchronized cohorts, 5 to 10 larvae per plate were randomly selected for measurement at 14, 26, 34 and 42 hours after the egg stage (correlating approximately to each of the four larval stages, L1-L4). At each time point, larvae were transferred to a glass slide containing a small NGM agar pad and immobilized with $10 \mu \mathrm{l}$ of $20 \mathrm{mM}$ sodium azide. Digital images were captured using a PixeLINK ${ }^{\mathrm{TM}}$ camera mounted on a Nikon Labophot and measurements were made using ImageJ (NIH, http://rsbweb.nih.gov/ij/). Larval growth was calculated as the difference in average length of animals between 14 and 42 hours after the egg stage, and converted to mm per hour.

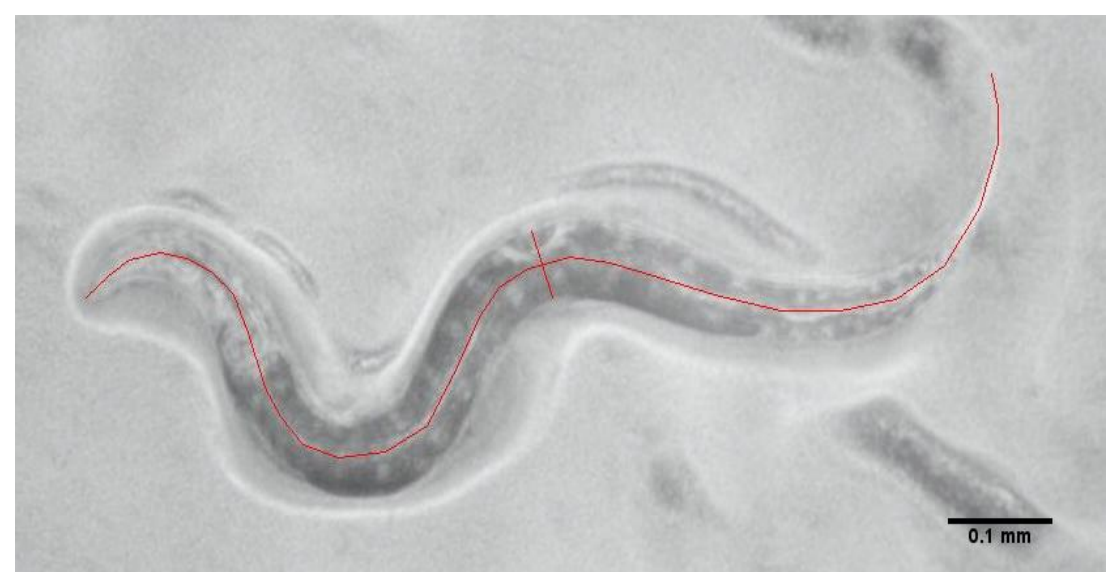

Figure 4. Caenorhabditis briggsae at fourth instar (L4). Lines for length and width measurements.

For adult measurements, all individual nematodes from the pharyngeal pumping assay were imaged live (on NGM plates) on days 0,6 and 12 from the L4 stage. Length measurements were made as above, as well as width at the vulva (Fig. 4), and volume was calculated following Bolanowski et al. (1981). Growth rates for length and volume were assessed between each time point and converted to mm per day. Note that, because 
only animals that survived to be measured on day 12 were used in our analyses, any laboratory selection on body size that may have been operating will not affect our results.

From the images captured to assess larval growth rates, larval stage at 42 hours after hatching was determined to assess the affect of the ND5 deletion on cell cycle progression in $C$. briggsae. Previous study of partial ETC disruption by increasing RNAi leading to life extension found a concomitant increase in delay of larval development rates, up to the point when life span was inversely affected (Rea et al., 2007). Here, ratios of L3 to L4 stage larvae at 42 hours after hatching were compared to the expected frequency (i.e. zero L3 larvae at 42 hours after hatching), and these ratios were compared between larvae from heteroplasmic and non-heteroplasmic isolates.

\section{Oxygen consumption}

The nature of the ND5 deletion, along with previous findings from other ETC complex I mutants, lead me to hypothesize that aerobic metabolic function will be compromised in C. briggsae isolates harboring higher frequencies of deletion-bearing mtDNA genomes. For whole-animal respiration rate quantification, production of massive, age-synchronized nematode cultures was achieved following Braeckman et al. (2002a). Oxygen consumption was measured on synchronous populations of L4 (L1+30 hours) animals using a Clark-type electrode following Braeckman et al. (2002a) except that data were normalized to the oxygen content of S-basal at $\sim 25^{\circ} \mathrm{C}$ as determined by an optical dissolved oxygen meter from Eureka Environmental Engineering. The protein 
content of samples was quantified using a BCA kit (Pierce) following the manufacturer's instructions after preparation following Braeckman et al. (2002a). Additionally, worm number per sample was calculated based on separately determined values of protein content per worm per isolate. Rates of oxygen consumption were normalized both to protein content and worm number.

\section{Statistics}

Statistical analyses were performed using R (R Development Core Team, 2009; MASS package by Venables and Ripley, 2002) and Microsoft Office Excel (2007). Not all variables are normally distributed; however, analysis of residuals from linear regression models indicate no outliers and Box Cox family transformations suggest that $\lambda=1$ (i.e. no transformation is able to improve the distribution) for all skewed variables. One-way analyses of variance were performed to determine variation between isolates per phenotype. To test for effects of heteroplasmy level on phenotypes, isolates were divided among three categories corresponding to relative ND5 deletion level (high, low, and none) and Tukey HSD pair-wise comparisons were made between these levels per trait ( $\alpha$ adjusted to correct for group-wise Type I error). This categorization enables graphical simplicity and reduces the potential effect on statistical results of high variation in heteroplasmy within isolates (see Howe and Denver 2008, Supplemental Table 1). Additionally, Spearman's rank correlation analyses were performed to test for relationships between isolate-specific ND5 deletion heteroplasmy and phenotypes. 


\section{RESULTS}

\section{Pharyngeal pumping}

Pharyngeal pumping rates over adulthood varied significantly between isolates on most days and were significantly negatively correlated to ND5 deletion until 14 days past the L4 stage (Table 1). For isolates categorized as having high levels of ND5 deletion heteroplasmy, pharyngeal pumping rates were significantly less than those with low or no heteroplasmy over days 2 through 18 (Tukey HSD p<0.05; high deletion isolates pumped

\section{Pharyngeal Pumping}

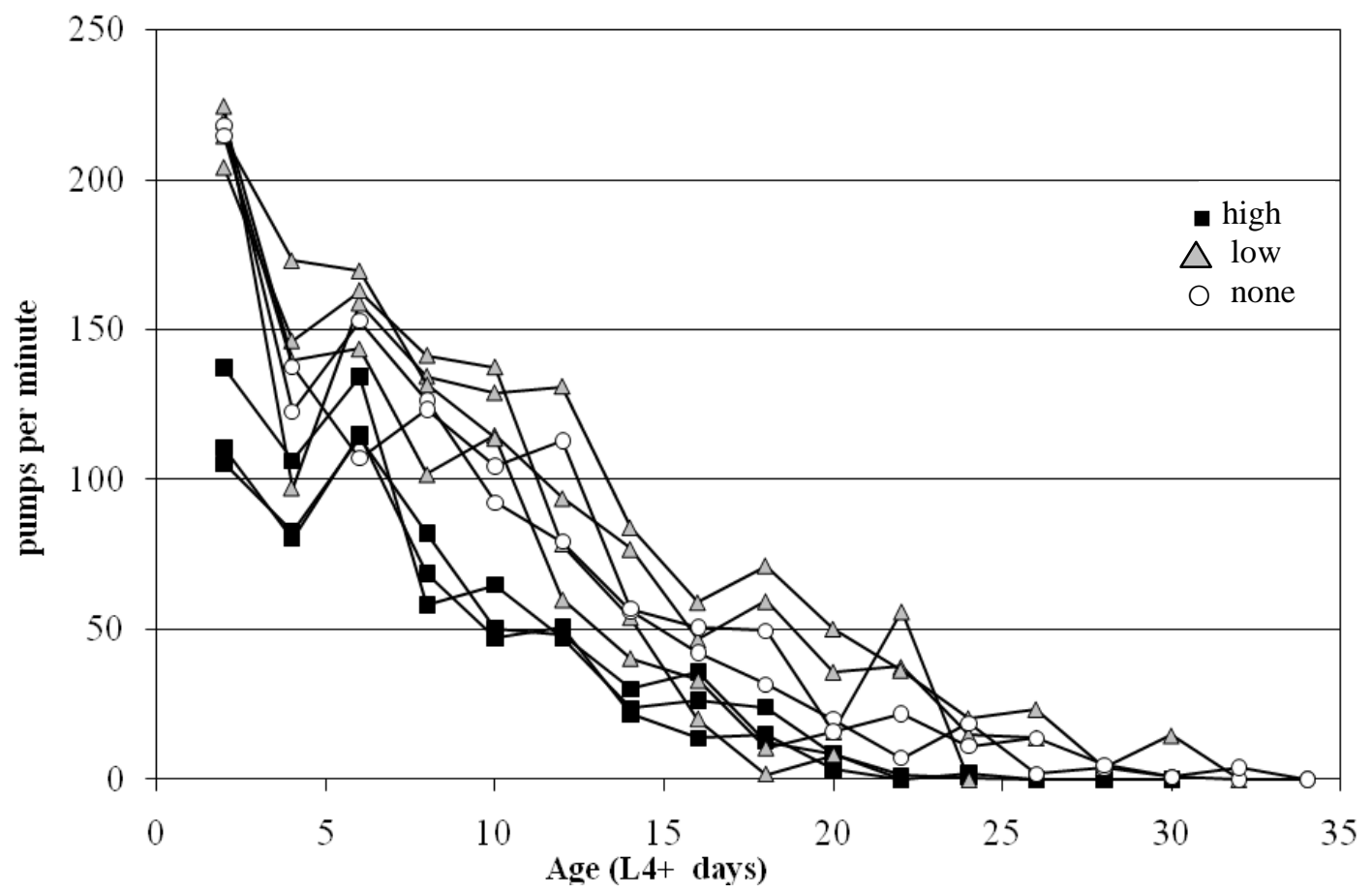

Figure 5. Adult pharyngeal pumping rates of $C$. briggsae. Natural isolate ND5 deletion heteroplasmy levels are indicated here as high (filled squares; HK105, HK104, VT847), low (grey triangles; PB800, EG4181, JU726, AF16), and none (clear circles; ED3092, ED3101). 
less than low- but not zero-deletion isolates on days 6 and 20; Fig. 5). The decline in pharyngeal pumping rate measured between 2 and 12 days or between 2 and 20 days post-L4 also varied significantly between isolates and these rates of change correlate negatively to ND5 deletion heteroplasmy (Table 1) such that high deletion isolates have a reduced rate of decline compared to low and no deletion isolates (Tukey HSD p<0.05). (Analysis of pharyngeal pumping ceased after day 20 due to decreased power from reduced sample sizes.)

\section{Larval Growth and Development}

Fourteen hours after hatching, larval length varied significantly between $C$.

briggsae isolates (Table 1) with individuals from high deletion isolates being longer than those from isolates with low or no deletion (Tukey HSD $\mathrm{p}<0.05$ ). Additionally, there was significant among-isolate variance in length at 26 and 34 hours after hatching (Table 1) and isolates without heteroplasmy were significantly shorter than isolates containing any amount of deleted genomes at these times points (Tukey HSD $\mathrm{p}<0.05$ ). Length of larvae at 42 hours after hatching was positively correlated to ND5 deletion heteroplasmy level (Table 1) such that all heteroplasmic isolates were longer than those with wildtype genomes only (Tukey HSD p<0.05; Fig. 6). Similarly, the rate of growth between 14 and 42 hours after hatching was significantly different among isolates (Table 1) such that heteroplasmic isolates grew at a faster rate between these two time points than did nondeletion-bearing isolates (Tukey HSD p<0.05). 
ND5 deletion-bearing isolates also developed faster than non-heteroplasmic isolates. A significant proportion of larvae at 42 hours were L3 instead of the expected L4 (40 of 312 larvae total, $\mathrm{p}<0.0001$ ). Further, significantly more of the 42-hour-old larvae from heteroplasmic isolates were L4 than were larvae from non-heteroplasmic isolates $(\mathrm{G}=3.16, \mathrm{df}=1, \mathrm{p}<0.05)$.

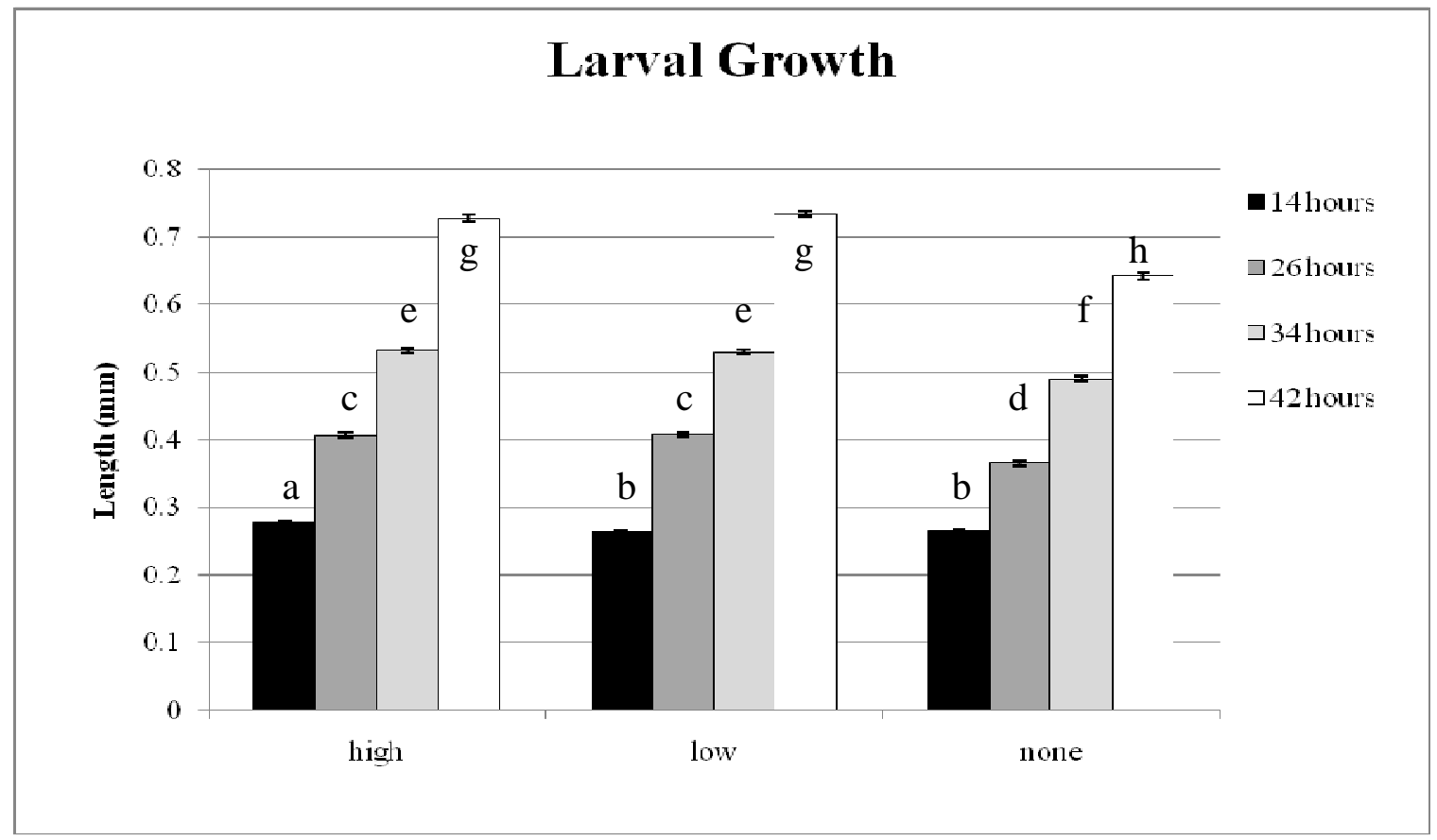

Figure 6. Length of $C$. briggsae larvae at 14, 26, 34, and 42 hours after hatching (which correspond approximately to L1, L2, L3, and L4). C. briggsae isolates are categorized by relative heteroplasmy levels; i.e., high (HK105, HK104, VT847), low (PB800, EG4181, JU726, AF16), and none (ED3092, ED3101), and the letters above bars indicate statistically indistiguishable groups (Tukey HSD, adjusted $\alpha=0.05$ ). Error bars indicate one standard error.

\section{Adult Growth}

At day 0 of adulthood (L4 stage), length varied significantly among isolates and was positively correlated to ND5 deletion frequency (Table 1). Volume exhibited the same relationship to ND5 deletion frequency (data not shown). Six days into adulthood, 
the same relationship was observed for length and volume (Table 1; volume data not shown): non-heteroplasmic isolates were significantly larger and exhibited a faster rate of growth between days 0 and 6 of adulthood compared to heteroplasmic isolates (Tukey HSD $\mathrm{p}<0.05)$. Twelve days after L4, C. briggsae harboring any level of ND5 deletion were significantly smaller than those without (length and volume, Tukey HSD p<0.05). In fact, heteroplasmic isolates exhibited almost no growth, or in some cases shrunk in length, between 6 and 12 days past L4, while non-deletion isolates continued to grow (Tukey HSD p<0.05; Fig. 7).

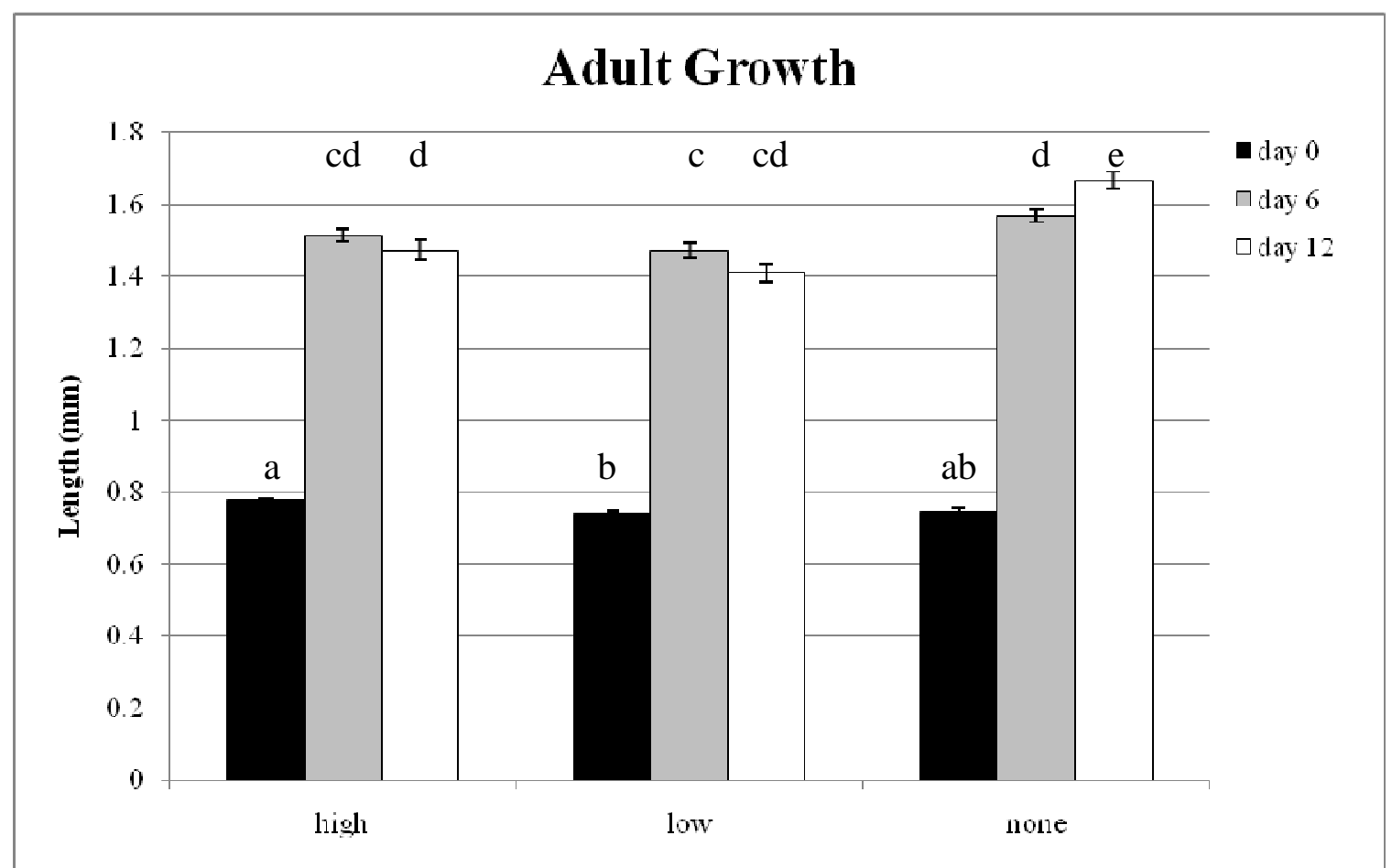

Figure 7. Length of $C$. briggsae adults at 0,6 , and 12 days post-L4. C. briggsae isolates are categorized by relative heteroplasmy levels; i.e., high (HK105, HK104, VT847), low (PB800, EG4181, JU726, AF16), and none (ED3092, ED3101), and letters above bars indicate statistically indistinguishable groups (Tukey HSD, adjusted $\alpha=0.05$ ). Error bars indicate one standard error. 


\section{Oxygen consumption}

Protein content per worm varied significantly among isolates $(F(8,7)=20.69$, $\mathrm{p}<0.001)$ but did not correlate to isolate-specific ND5 deletion heteroplasmy levels (Spearman's $\left.\mathrm{r}_{\mathrm{s}}=0.09, \mathrm{p}=0.74\right)$. Similarly, oxygen consumption rates varied significantly among isolates (per mg protein: $F(8,9)=6.54$, $p<0.01$; per worm: $F(7,8)=18.18$, $p<0.001$ ) but did not correlate to isolate-specific heteroplasmy levels (per mg protein: $r_{s}=-0.34$, $p=0.17$, Fig. 6; per worm: $r_{s}=0.06, p=0.83$ ). (Oxygen consumption rates in Fig. 8 are normalized to protein content, as is the convention for C. elegans; see discussion in Braeckman et al., 2002b.)

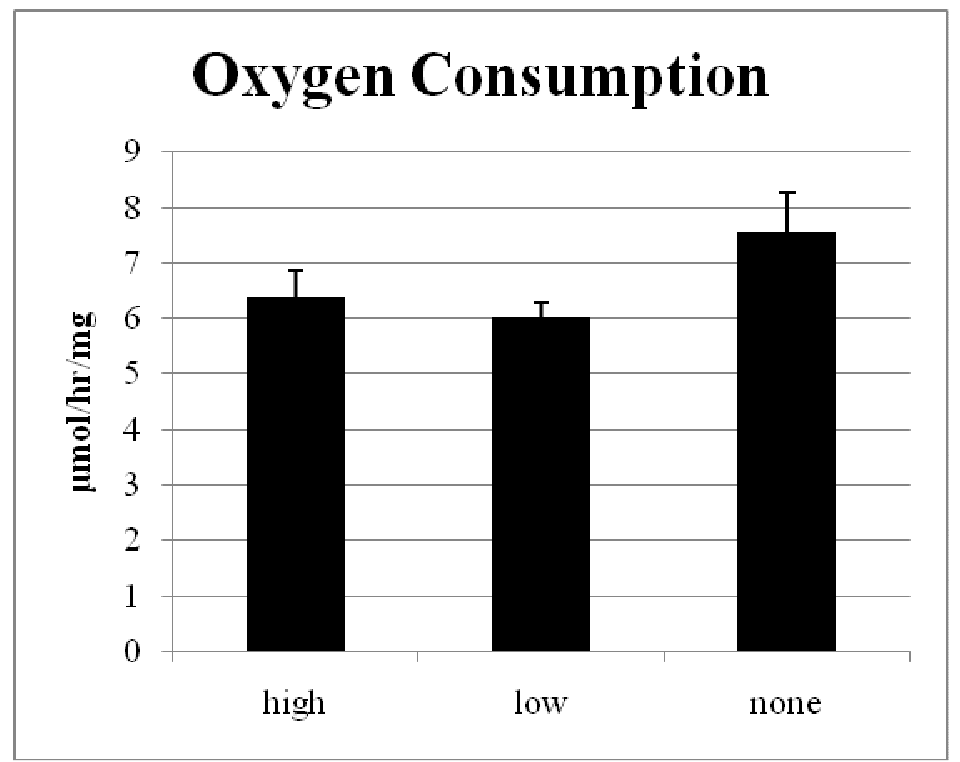

Figure 8. Whole-organism rates of oxygen consumption of $C$. briggsae. Isolates categorized by relative heteroplasmy levels; i.e., high (HK105, HK104, VT847), low (PB800, EG4181, JU726, AF16), and none (ED3092, ED3101). Error bars indicate one standard error. 
Chapter 1

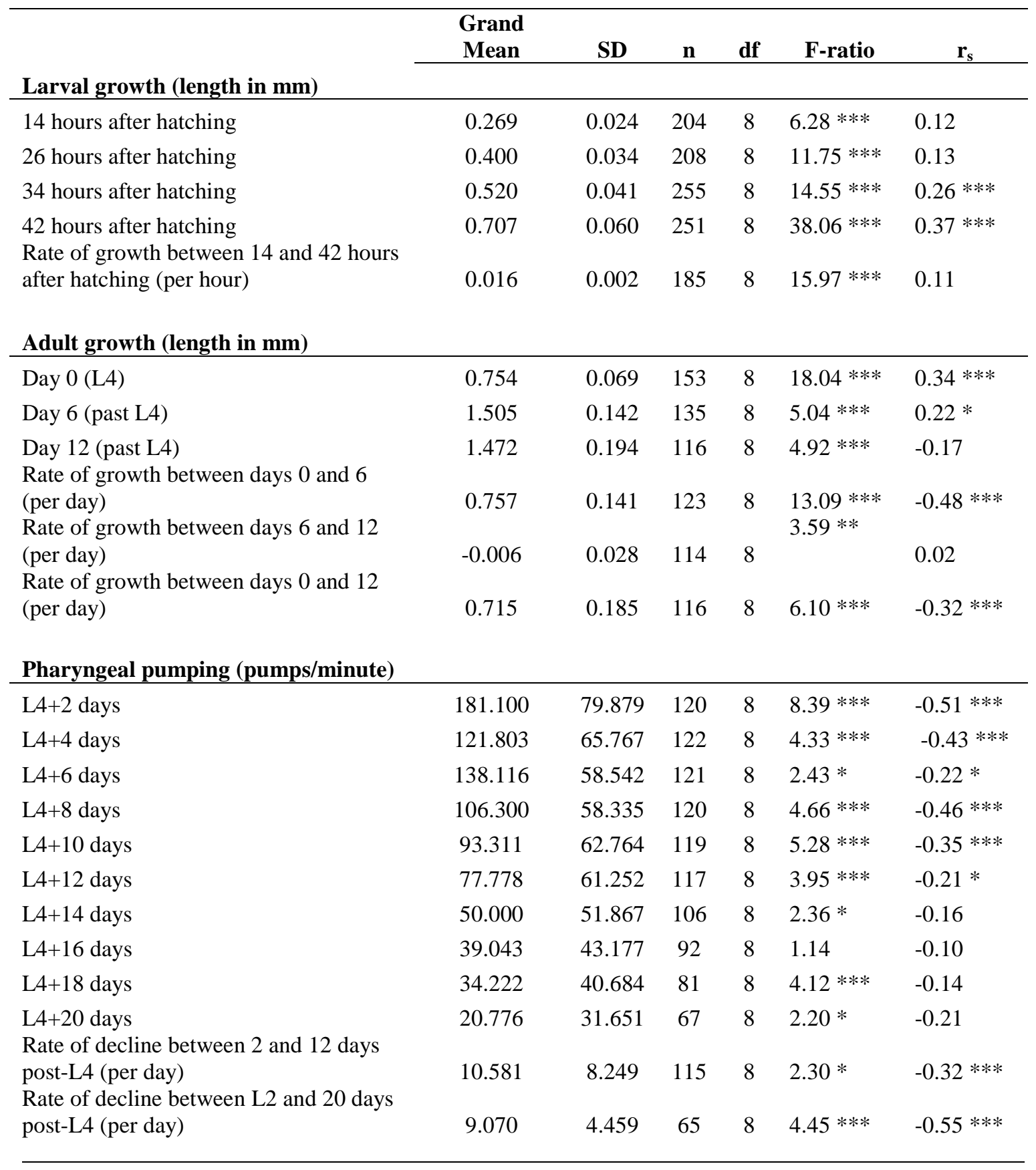

Table 1. Test statistics per phenotype measured among nine natural isolates of Caenorhabditis briggsae. $\left(r_{\mathrm{s}}\right.$ is the Spearman's rank correlation between isolate-specific trait means and deletion frequencies. *, **, and $* * *$ denote significance at $\alpha=0.05,0.01$, and 0.001 , respectively.) 


\section{DISCUSSION}

To evaluate the effects of a naturally occurring mtDNA deletion in the nematode C. briggsae, several aging and physiology-related phenotypes have been measured and compared among natural isolates chosen to capture the full range of ND5 deletion heteroplasmy level in this species.

\section{Pharyngeal pumping}

Deterioration of the pharynx muscles and decline in pharyngeal function have previously been correlated to aging in $C$. elegans (Collins et al., 2008). Here, C. briggsae isolates harboring more ND5 deletion-bearing mitochondrial genomes were shown to have significantly decreased rates of pharyngeal pumping over much of adulthood when compared to isolates with low levels of deleted genomes or none at all. Interestingly, the rate of decline in pharyngeal pumping is lower for high-deletion isolates than for others.

These results suggest that, although $C$. briggsae harboring more deleted ND5 subunits are perhaps more aged over adulthood than those with fewer or no deleted ND5 products, the former are aging less rapidly.

The relationship between heteroplasmy and pharyngeal pumping in C. briggsae does not align with the non-linear relationship between heteroplasmy and in vivo superoxide production found by Estes et al. (in review). However, when considered alongside exogenous oxidative stress resistance, the disparity in between-isolate 
differences of pharyngeal pumping rates and superoxide production may be explained. That is, C. briggsae present as less aged via either decreased superoxide production (as in the low-heteroplasmy isolates) or increased oxidative stress resistance (as in the nonheteroplasmic isolates). This interpretation mimics somewhat the 'hormetic effect' seen between exogenous oxidative stress and upregulation of certain oxidant scavenging mechanisms seen in C. elegans and proposed by Rea (2005) as one possible mechanism for life extension in certain C. elegans 'mit mutants.' Additionally, these results imply that a threshold effect for this particular deletion may be at play since high heteroplasmic C. briggsae may be less equipped to overcome the levels of superoxide associated with high levels of the ND5 deletion. Considering that $C$. briggsae heteroplasmy does not exceed around 50\% in all natural isolates studied (see Howe and Denver, 2008), perhaps eggs or larvae with greater than $50 \%$ deleted genomes are inviable due to a greater lack of tolerance combined with even higher elevated ROS levels compared to the highest deletion-bearing isolates studied here. However, without longitudinal study of stress resistance and superoxide production among $C$. briggsae isolates, the cause of variation in the rate of decline of pharyngeal pumping found here cannot be explained.

\section{Growth}

Growth rates for $C$. briggsae larvae vary markedly between isolates and correlate positively to relative amounts of heteroplasmy. Interestingly, C. briggsae larvae with any amount of partially-deleted genomes grow more rapidly and to a greater mean length than 
do larvae from non-heteroplasmic isolates. These findings contradict patterns previously observed in C. elegans where increased RNAi of various ETC-associated genes was accompanied by decreased larval development and earlier developmental arrest (Rea et al., 2007). This relationship was observed at levels of RNAi dilution that first triggered increased longevity, and continued as increasing interference worked to decrease longevity (Rea et al., 2007). The enhanced larval development in high ND5 deletion $C$. briggsae isolates could indicate a change in life history strategy due to elevated superoxide production leading to decreased lifespan (Estes et al., in review). However, confounding such an interpretation is the same significantly increased larval growth in low heteroplasmic $C$. briggsae that produce the least amount of superoxide and live longest compared to non heteroplasmic isolates (Estes et al., in review).

The reverse relationship between growth and heteroplasmy is seen over adulthood in $C$. briggsae, thus coinciding with previous results for $C$. elegans with ETC disrupted via RNAi (Dillon et al., 2002; Rea et al., 2007). Non-heteroplasmic isolates continue to grow in length between 6 and 12 days past maturation (as in C. elegans, see Croll et al., 1977 and Bolanowski et al., 1981) while heteroplasmic C. briggsae tend to shrink. Given that $C$. elegans aging is marked with a progressive decrease in muscle cell organization, loss of cell structure, and cell shrinkage due to loss of cytoplasm (Collins et al., 2008), the later change in adult size of heteroplasmic $C$. briggsae is another indication that progressive aging accompanies higher levels of ND5 deletion heteroplasmy. 
The stark change in $C$. briggsae growth rate correlates to the switch from heavily anaerobic to aerobic metabolic pathways between larval and adult stages (Braeckman et al., 2008). In wild-type C. elegans, entry to L4 is marked by a 3-fold increase in mtDNA copy number while another 16-fold increase occurs at the final molt from L4 to adult (Bratic et al., 2009). Although these grand expansions of C. elegans mitochondrial genomes are tightly coupled to germline cell proliferation and embryogenesis, $C$. briggsae isolates harboring high ND5 deletion heteroplasmy may suffer when relying on the aerobic ETC pathway during adulthood especially considering declines of maternallyprovided factors in adults (i.e. mRNA; see Rea, 2005). Combined with the decline in pharyngeal pumping, adult growth rates imply that a high degree of heteroplasmy in $C$. briggsae denotes advanced aging in adulthood.

\section{Oxygen consumption}

Although oxygen consumption rates of $C$. briggsae L4 vary significantly among isolates, there exists no correlation to relative or isolate-specific heteroplasmy levels. This lack of correlation may be due to the age at measurement here which coincides with the change from primarily anaerobic metabolism in larvae to aerobic respiration in adults (Braeckman et al., 2008). An additional confounding factor to analysis of the oxygen consumption measures reported here is the knowledge that different $C$. briggsae isolates develop at different rates (see above). Therefore, although each measure of oxygen consumption was performed on massive age-synchronized populations after the same 
length of time cultured ( \pm 1 hour), the values may reflect rates of oxygen consumption of C. briggsae at different ages. True evaluation of aerobic respiratory capacity of $C$. briggsae via oxygen consumption will require longitudinal measures taken well before, during and after metamorphosis into adulthood. 


\section{CHAPTER 2}

Inter-Population Hybrids of Caenorhabditis briggsae Natural Isolates

\section{BACKGROUND}

As the primary energy producers in most eukaryotic cells, mitochondria are critical for the survival of most organisms and their dysfunction can lead to inefficient energy metabolism, disease, aging, and early death (Chinnery and Turnbull, 2000; DiMauro and Schon, 2003; Smeitink et al., 2003; Taylor and Turnbull, 2005; Wallace, 2005). Across many taxa, strong correlations have been drawn between mitochondrial function, and aging and longevity; however, a causal link between mitochondrial function and aging has yet to be demonstrated (Rea and Johnson, 2003; Balaben et al., 2005 Rea, 2005; Lee and Wei, 2007; Hiona and Leeuwenburgh, 2008; Gems and Doonan, 2009; Lapointe and Hekimi, 2010). A major hurdle to understanding the role of mitochondria in aging and disease within an evolutionary or population genetic context has been the lack of model systems containing relevant natural mitochondrial genetic variation. In the nematode Caenorhabditis briggsae, the occurrence of a geographically segregating heteroplasmic mutation affecting a key unit of complex I of the electron transport chain (ETC) provides a novel chance for examining the relationship between mitochondrial 
dysfunction, longevity and disease without the use of mutagens, genetic manipulation, or cell culture manipulation.

In several recently isolated populations of $C$. briggsae, a deletion removing nearly half of the mitochondrial encoded $N A D H$-dehydrogenase 5 (ND5) protein coding gene has been discovered (Howe and Denver, 2008; Fig. 1). When present, the partial deletion of ND5 in C. briggsae occurs in a heteroplasmic state where some portion of mitochondrial genomes harbor the deletion (mutant) and the rest do not (wildtype). Among C. briggsae isolates, heteroplasmy of the ND5 deletion ranges from 0 to $50 \%$ (Fig. 2). Although the mutant ND5 gene in C. briggsae is transcribed, as evidenced by identification of truncated mRNA sequences, its degree of functionality is unknown. However, because the ND5 gene product likely functions as a proton translocator in the distal part of the membrane bound domain of complex I, and because the ND5 subunit is rate limiting to complex I assembly, the ND5 deletion is likely to have a severe negative impact on complex I efficiency in C. briggsae. In support of this idea, several neurodegenerative diseases have been associated with mutations in ND5 and other complex I subunits (Janssen et al., 2006).

Reactive oxygen species (ROS) are normal byproducts of complex I activity and have been suspected as a key determinant of aging and longevity due to their highly destructive properties. Based on studies of other complex I mutants (Pitkanen and Robinson, 1996; Luo et al., 1997; Bai et al., 2000; Kayser et al., 2004; Janssen et al., 2006; Park et al., 2009), the partial ND5 deletion in C. briggsae is suspected to lead to 
elevated levels of ROS, perhaps such that ROS will increase with heteroplasmy levels. Once heteroplasmy and ROS levels in C. briggsae reach levels beyond those that can be remedied via natural oxidant defense mechanisms, heteroplasmy is suspected to negatively impact lifespan and aging in $C$. briggsae. To establish $C$. briggsae as a natural model for studying the process of aging and lifespan determination, several life history characteristics have been evaluated for various isolates from around the world spanning the range of heteroplasmy in this species. Total offspring production was shown to correlate negatively with heteroplasmy in C. briggsae (Howe and Denver, 2008) as was lifespan and population growth rates (Estes et al., in review). Interestingly, neither ATP content nor in vitro hydrogen peroxide (a form of ROS) production exhibit a significant relationship to deletion heteroplasmy level, yet oxidative stress resistance and in vivo superoxide (another ROS) production do (Estes et al., in review). In Chapter 1 of this thesis, high isolate-specific heteroplasmy levels in C. briggsae were associated with increased rates of larval development and aging and decreased adult growth. Overall, these finding suggest that ND5 deletion heteroplasmy in C. briggsae does impact life history and may indicate an adaptive response via more rapid development to adulthood in order to minimize damage from ROS prior to reproduction.

To establish that the phenotypic variance observed among natural isolates of $C$. briggsae is due in fact to ND5 deletion heteroplasmy rather than to natural nuclear genetic variation segregating among isolates, several inter-population hybrid lines of $C$. briggsae have been created from natural isolates with high and low heteroplasmy levels. 
After nine generations of crossing hybridized offspring back to males of the nuclear genetic source population, hybrids were considered to harbor only mitochondrial genetic information from one population on the complete nuclear genetic background of another population, resulting in $>99 \%$ 'mitochondrial replacement.' Assuming the maternal inheritance of mitochondria and that natural ND5 deletion heteroplasmy controls the phenotypic variation previously observed among natural C. briggsae isolates, and barring epistasis between genomes that control the measured traits, hybrid trait means should closely match those for each parental source of mitochondria.

Somewhat contrary to expectations, for most traits measured, hybrid C. briggase differ not only from their parental populations but also from other replicates of the same introgressions. Surprisingly, mitochondrial genotyping of C. briggsae hybrid lines revealed that mitochondria were not inherited through the maternal lineage in most cases. However, inspection of results from hybrids in which maternal transmission of mitochondria was successful indicate that epistasis may be important for most traits measured here, such that conflict between mitochondrial and nuclear genes that have evolved separately in different populations leads to breakdown of fitness and other traits in these hybrids; i.e., cytonuclear conflict. 


\section{METHODS}

\section{Nematode Strains and Maintenance}

In order to disentangle competing effects of mitochondrial versus nuclear genetic variation on the among-isolate phenotypic differences reported in Chapter 1, C. briggsae experimental lines were generated to allow me to assess the effects of a mitochondrial genome on different nuclear genetic backgrounds. Hybrid experimental lines were created by reciprocally crossing two pairs of $C$. briggsae natural isolates. Each cross utilized one parental isolate with high ND5 deletion heteroplasmy level and one with low heteroplasmy level (Fig. 9). (Natural isolates were provided by D. R. Denver, OSU.). Three replicate introgressions were performed for each pair of natural isolates, resulting in a total of 12 hybrid $C$. briggsae lines (Table 2). Nematodes were grown under standard laboratory conditions at $25^{\circ} \mathrm{C}$ on $15 \mathrm{~mm}$ NGM plates and Escherichia coli strain $\mathrm{HB} 101$, unless otherwise noted.

\section{Nematode Hybridization}

Each replicate of the four $C$. briggsae introgressions was initiated by crossing one egg-laden hermaphrodite from the mitochondrial source isolate with 5-10 males from the nuclear source isolate for a given cross. Multiple males were paired with each hermaphrodite to ensure successful outcrossing. Male-rich colonies were maintained by manual enrichment of source populations every other day. Males were removed the next 
day to avoid damage to hermaphrodites from excessive mating. Three days after the initial cross, plates were examined for successful male fertilization as indicated by a 1:1 offspring sex ratio. Once offspring had reached approximately the L4 stage, one hermaphrodite from a successful cross was transferred to a new plate and crossed with 510 males from the nuclear source population. (Typically, more than one hybrid hermaphrodite was isolated per replicate and crossed with males in this manner to ensure advancement to the next outcrossing since numerous hybrid lineages went extinct.) The nuclear DNA (nDNA) of hybrid offspring should be entirely replaced after nine successful matings of hybrid hermaphrodites to males of the nuclear source population. In other words, F10 hybrid individuals should carry only mitochondrial DNA (mtDNA) from one natural $C$. briggsae isolate and only nDNA from a second isolate.

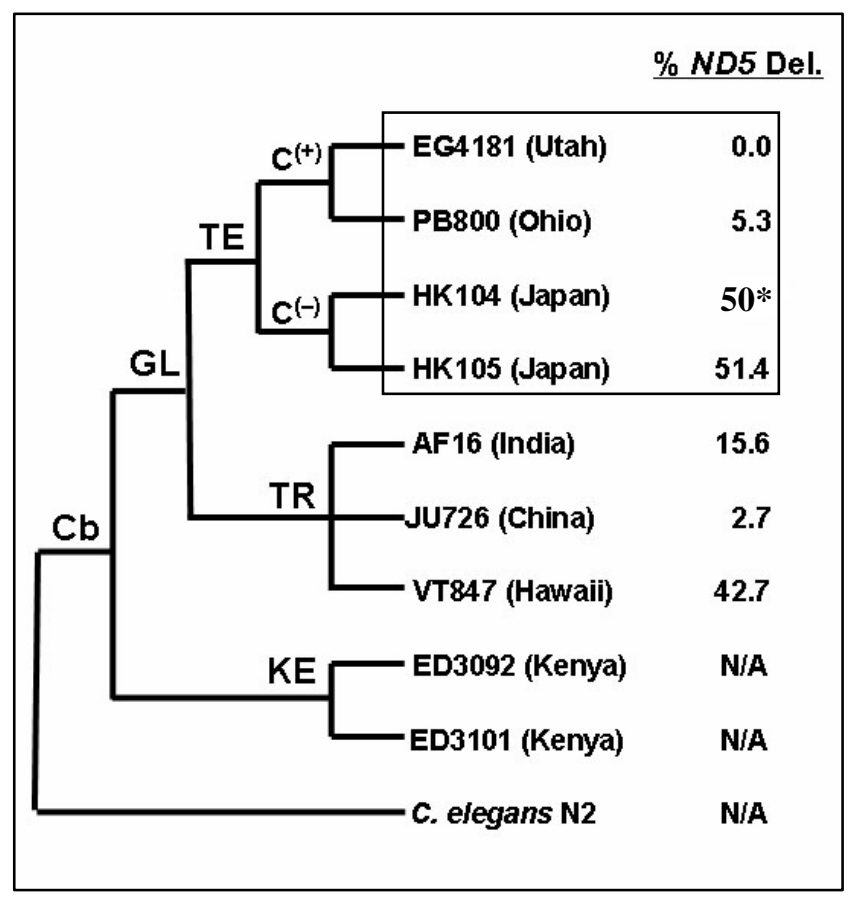

Figure 9. C. briggsae natural isolates selected for hybridization. Two pairs of high and low heteroplasmy isolates (outlined on this intraspecific phylogeny where \% ND5 Del. indicates isolate-specific percentages of total mitochondrial genomes that harbor ND5 deletions, as determined by qPCR (Howe and Denver, 2008)) were used as progenitors for hybrid lines. $(\mathrm{GL}=$ global intraspecific superclade; $\mathrm{KE}=$ Kenya clade; TE and $\mathrm{TR}=$ temperate and tropical subclades of GL. $\mathrm{C}(+)=$ temperate-clade isolates bearing compensatory $\Psi$ ND5-2 alleles; $\mathrm{C}(-)=$ those bearing ancestral alleles.) * The HK104 strain used here was the inbred progenitor of mutationaccumulation lines (Baer et al., 2005) found to harbor high ND5 deletion levels similar to those of HK105 (Dee Denver, pers. comm.). 
Fitness and Lifespan

The partial ND5 mtDNA deletion segregating among natural $C$. briggsae isolates has previously been negatively correlated to fitness (Howe and Denver, 2008). To establish whether this relationship is in fact due to the natural mtDNA variation among isolates rather than to nDNA variation among isolates, offspring production and lifespan of hybrid C. briggsae mitochondrial replacement lines were evaluated and compared to the parental lines.

For each parental natural isolate and replicate introgressed line, several adult hermaphrodites were placed on Petri dishes, allowed to lay eggs for three hours and then removed. Two days later, 10-20 L4 individuals per line were transferred to a new plate each, and again $\sim 24$ hours later over the duration of the reproductive period. After each transfer, plates remained at room temperature for $\sim 24$ hours to allow viable eggs to hatch;

\begin{tabular}{lcccc}
\hline Mt Origin & $\begin{array}{c}\text { Nuclear } \\
\text { Origin }\end{array}$ & Rep & Annotation & $\begin{array}{c}\text { Expected ND5 } \\
\text { deletion \% }\end{array}$ \\
\hline \multirow{3}{*}{ HK104 } & \multirow{2}{*}{ EG4181 } & 1 & mHnE_1 & \\
& & 3 & mHnE_2 & $\sim 50$ \\
& & 1 & mEnH_3 & \\
EG4181 & HK104 & 2 & mEnH_2 & $\sim 0$ \\
& & 3 & mEnH_3 & \\
\hline \multirow{3}{*}{ HK105 } & PB800 & 1 & mHnP_1 & \\
& & 3 & mHnP_2 & $\sim 50$ \\
\hline \multirow{3}{*}{ PB800 } & HK105 & 1 & mPnH_3 & \\
& & 3 & mPnH_2 & $\sim 5$ \\
\hline
\end{tabular}

Table 2. Expected ND5 deletion heteroplasmy levels in C. briggsae hybrids. In crossing C. briggsae natural isolates with high and low ND5 deletion levels, these expectations are based on maternal natural isolate heteroplasmy ( $c f$. Fig. 9). 
then $1 \mathrm{~mL}$ of $0.3 \mathrm{M}$ sodium azide per plate was added to euthanize larvae. Plates of progeny were stored at $4{ }^{\circ} \mathrm{C}$ until later enumeration which was enabled by staining $E$. coli and agar with $0.075 \%$ toluidine blue. Total lifespan of adult hermaphrodites was determined as the number of days lived since hatching.

\section{Pharyngeal pumping}

Pharyngeal pumping rate is known to decline steadily with age in C. elegans and, therefore, serves as a reliable biomarker of age (Collins et al., 2008). ND5 deletion heteroplasmy in natural $C$. briggsae isolates has been found to correlate negatively to adult pharyngeal pumping (Chapter 1 of this thesis), implying that increased levels of the partial ND5-deletion may affect rates of aging in C. briggsae. If, indeed, the ND5 deletion alters rates of aging and it is maternally transmitted to offspring as expected, pharyngeal pumping in $C$. briggsae hybrids should - barring cytonuclear epistasis closely match that for the natural $C$. briggsae isolate that contributed the mtDNA.

To compare rates of aging between hybrid replicates and parental lineages, pharyngeal pumping was assessed every other day during adulthood starting at two days past L4 (fourth instar). The same 10-20 individuals per line analyzed in the fitness and longevity assays were examined for pharyngeal pumping, except for the third replicate of each introgressed line. Pharyngeal pumping assays were performed at room temperature on NGM plates and calculated as the average number of terminal bulb pumps from three 
5-second intervals, converted to pumps per minute. Decline in pharyngeal pumping rates were calculated between days 2 and 12 .

\section{Growth}

As with pharyngeal pumping, if the partial deletion of ND5 is responsible for variation in larval and adult growth between natural isolates of $C$. briggsae, hybrid growth rates should match those for the relevant mitochondrial source population. For larval measurements, nematodes were age synchronized by allowing 10-20 gravid hermaphrodites per isolate and hybrid replicate to lay eggs on fresh $15 \mathrm{~mm}$ NGM plates with E. coli OP50-1 for two hours. From these age-synchronized cohorts, 5 to 10 larvae per plate were randomly selected for measurement at 14, 26, 34 and 42 hours after the egg stage (correlating approximately to each of the four larval stages, L1-L4). At each time point, larvae were transferred to a glass slide containing a small NGM agar pad and immobilized with $10 \mu \mathrm{l}$ of $20 \mathrm{mM}$ sodium azide. Digital images were captured using a PixeLINK $^{\mathrm{TM}}$ camera mounted on a Nikon Labophot and measurements were made using ImageJ (NIH, http://rsbweb.nih.gov/ij/). Larval growth was assessed as the difference in the average lengths of animals between 14 and 42 hours after the egg stage, and converted to $\mathrm{mm}$ per hour.

For adult measurements, all individual nematodes from the pharyngeal pumping assay were imaged live (remained on NGM plates) on days 0, 6 and 12 from the L4 stage. Length measurements were assessed as above (Fig. 4) and growth rates in length were 
calculated between 0 and 6 , and 0 and 12 days then converted to mm per day. Note that, because only animals that survived to be measured on day 12 were used in this analyses, any laboratory selection on body size that may have been operating will not affect our results.

From the images captured to assess larval growth rates, larval stage at 42 hours after hatching was determined to assess the affect of introgression and inheritance of the mtDNA deletion in $C$. briggsae on cell cycle progression in $C$. briggsae. Ratios of L3 to L4 stage larvae at 42 hours after hatching were compared to the expected frequency (i.e. zero L3 larvae at 42 hours after hatching), and these ratios were compared between hybrid lineages and parental isolates.

\section{Oxygen consumption}

Whole-organism oxygen consumption was previously measured on age synchronized samples and compared to determine whether natural mtDNA variation affects aerobic respiration among C. briggsae natural isolates (Chapter 1 of this thesis). Oxygen consumption rates were found to vary significantly between natural isolates but did not correlate significantly to ND5 deletion heteroplasmy. To determine whether this variation is due to the mtDNA variation between isolates, oxygen consumption rates of hybrid $C$. briggsae were measured and evaluated against values for the natural mitochondrial source population of each introgression. 
Whole-animal respiration rates were measured according to Braeckman et al. (2002a) with some modification, and these data were normalized to the protein content per sample using a BCA kit (Pierce) following preparation described in Braeckman et al. (2002a). Data were obtained for only two replicates of each introgression, except mHK105 x nPB800 for which all replicates were measured.

\section{Statistics}

Statistical analyses were performed using R (R Development Core Team, 2009; MASS package by Venables and Ripley, 2002) and Microsoft Office Excel (2007). Differences between parental isolates were assessed using t-tests for each trait. All replicates of each introgression were compared using one-way analyses of variance for each phenotype, or t-tests when applicable. Finally, hybrid replicates from each introgression were pooled and compared to the appropriate maternal isolate using a t-test.

\section{Genotyping}

To verify maternal transmission of mitochondrial genomes and assess the ND5 deletion heteroplasmy level in hybrid C. briggsae, molecular analyses of hybrid and parental strains were performed by collaborator D. K. Howe at the Center for Genome Research and Biocomputing (OSU, Corvallis, OR). Briefly, a published PCR assay (Howe and Denver, 2008) was used to assess the relative frequency of the ND5 deletion in each replicate of all introgressions and each parental isolate. These analyses were 
performed twice for each replicate of each hybrid lineage in Table 2. Additionally, sequencing of microsatellite loci within the mitochondrial cytochrome-oxidase II (COII) gene was conducted to verify maternal inheritance of mtDNA genomes. 


\section{RESULTS}

Several lines of $C$. briggsae hybridized from natural isolates with high and low ND5 deletion heteroplasmy were created in order to determine whether the phenotypic variance observed among natural isolates is due to mitochondrial genetic differences among those isolates. If so, and assuming that mitochondria are inherited maternally and ND5 deletion frequency is transmitted to offspring, hybrid trait means should closely match those for each respective maternal progenitor.

Fitness

Daily fecundities from the parental and reciprocal hybrid strains from crossing $C$. briggsae natural isolates EG4181 and HK104 are shown in Figure 10. Fitness varied significantly between EG4181 and HK104 natural isolates (low and high heteroplasmy, respectively) with the former producing significantly more offspring overall $(\mathrm{t}(58)=-$ 12.19, $\mathrm{p}<0.001$; Table 3). The mHK104 x nEG4181 (mHnE from here on, as in Table 2) hybrids also varied significantly between replicates in total offspring production $\left(\mathrm{t}(32)=7.58, \mathrm{p}<0.001\right.$; data unattainable for the third replicate, $\mathrm{mHnE} \_3$, due to lack of viable larvae from age-synchronized populations; Table 4). When pooled, mHnE hybrids produced significantly more offspring than the maternal source, HK104 $(\mathrm{t}(52)=4.19$, $\mathrm{p}<0.001$; Table 4). Hybrids of mEG4181 x nHK104 (mEnH from here on, as in table 2) derivation did not vary significantly among replicates $(F(2,29)=2.12, p=0.14$; Table 5) 
but produced significantly less offspring when pooled than the maternal source, EG4181 $(\mathrm{t}(70)=-6.32, \mathrm{p}<0.001$; Table 5). (Note that progeny production on the fifth day of maturity was not obtained for the third replicate of each introgression.)

Daily fecundities of the parental and reciprocal hybrid strains from crossing $C$.

briggsae natural isolates PB800 and HK105 are shown in Figure 11. Low heteroplasmy natural C. briggsae isolate PB800 produced significantly more offspring overall than high heteroplasmy isolate HK105 (t(48)=-6.42, p<0.001; Table 3). mHK105 x nPB800 hybrids (mHnP from here on, as in Table 2) varied significantly among replicates for this

\section{Reproductive Schedule}

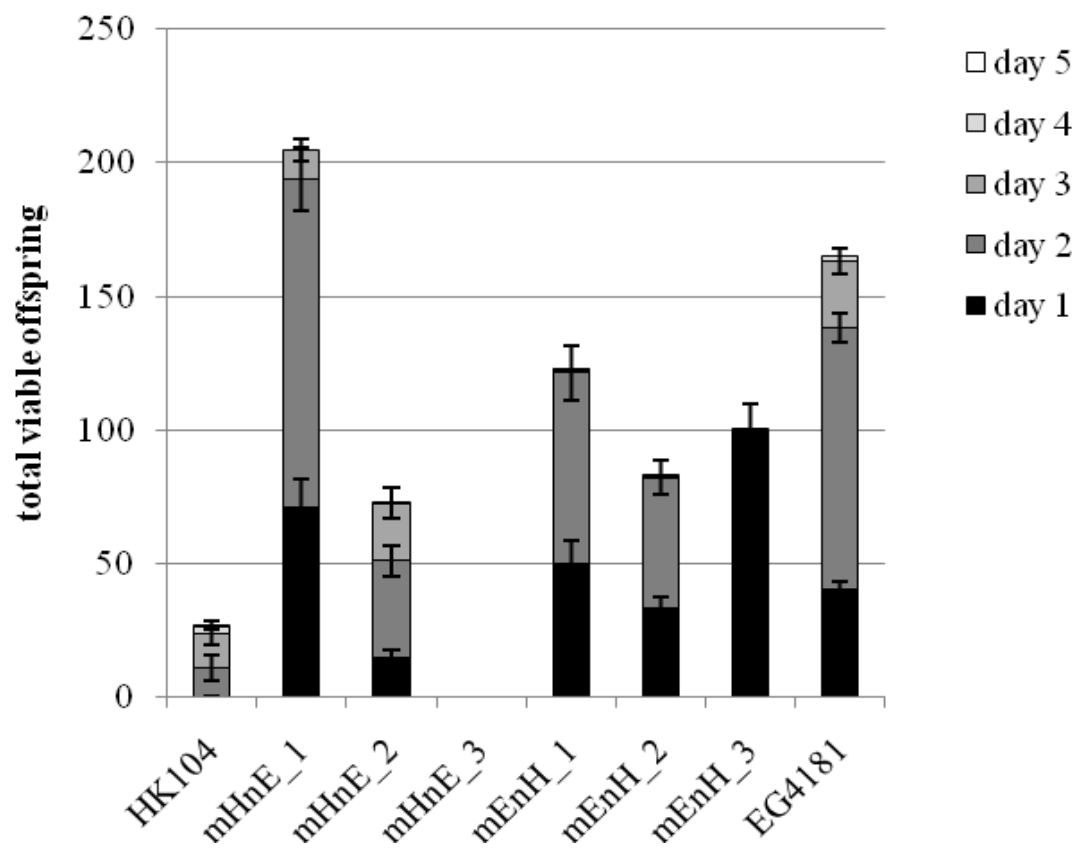

Figure 10. Total reproductive output for each parental and introgressed line of natural C. briggsae isolates HK104 (high heteroplasmy) and EG4181 (low heteroplasmy). Total progeny per day of reproduction is indicated by bar color as denoted in the figure legend. Error bars indicate one standard error. (Data missing for $\mathrm{mHnE} \_3$ - see text.) 
trait $(\mathrm{F}(2,31)=8.50, \mathrm{p}<0.01$; Table 6$)$ and, when pooled, produced significantly more offspring than the putative mitochondrial source population, isolate HK105 $(\mathrm{t}(52)=5.86$, p<0.001; Table 6). However, C. briggsae hybrids mPB800 x nHK105 (mPnH from here on, as in Table 2$)$ did not vary significantly among replicates $(\mathrm{F}(2,41)=1.79, \mathrm{p}=0.18$; Table 7) and produced less total offspring when pooled and compared to their maternal source, PB800 $(\mathrm{t}(72)=-4.93, \mathrm{p}<0.001$; Table 7$)$. (Note that progeny produced on the fifth day of maturity was not obtained for the third replicate of each introgression.)

\section{Reproductive Schedule}

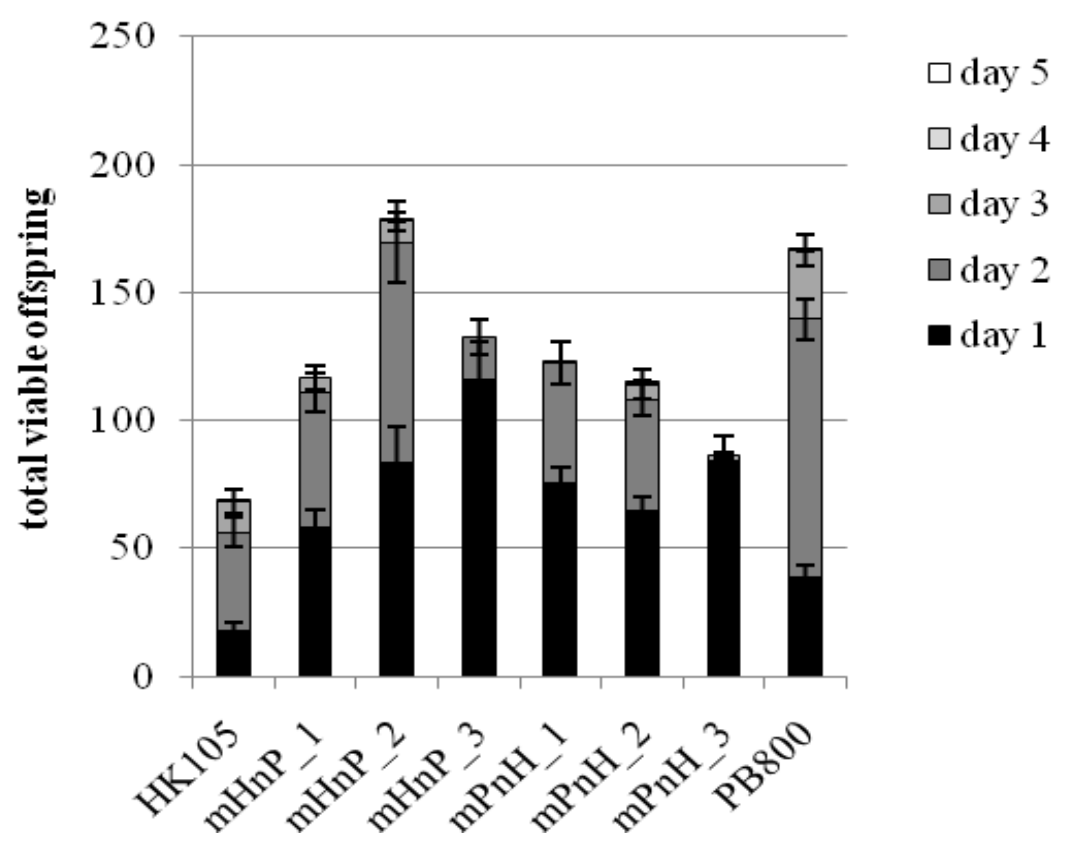

Figure 11. Total reproductive output for each parental and introgressed line of natural C. briggsae isolates HK105 (high heteroplasmy) and PB800 (low heteroplasmy). Total progeny per day of reproduction is indicated by bar color as denoted in the figure legend. Error bars indicate one standard error. 
Lifespan

Lifespan of the parental and reciprocal hybrid strains from crossing C. briggsae natural isolates EG4181 and HK104 are shown in Figure 12. Lifespan in days since hatching does not vary significantly between $C$. briggsae natural isolates HK104 and EG4181 $(\mathrm{t}(43)=-1.62, \mathrm{p}=0.11$; Table 3$)$. C. briggsae hybrid mHnE longevity did not differ significantly between replicates $(\mathrm{t}(16)=0.32, \mathrm{p}=0.76$; data were unattainable for the third replicate, mHnE_3, due to lack of viable larvae from age-synchronized populations) or from the maternal source natural isolate, HK104, when pooled $(\mathrm{t}(38)=1.20, \mathrm{p}=0.24$; Table 4). Similarly, mEnH hybrids did not differ significantly in lifespan either among replicates of this particular introgression $(\mathrm{F}(2,26)=2.17, \mathrm{p}=0.13)$ or when pooled and compared to the maternal source isolate, EG4181 $(\mathrm{t}(47)=-1.62, \mathrm{p}=0.11$; Table 5).

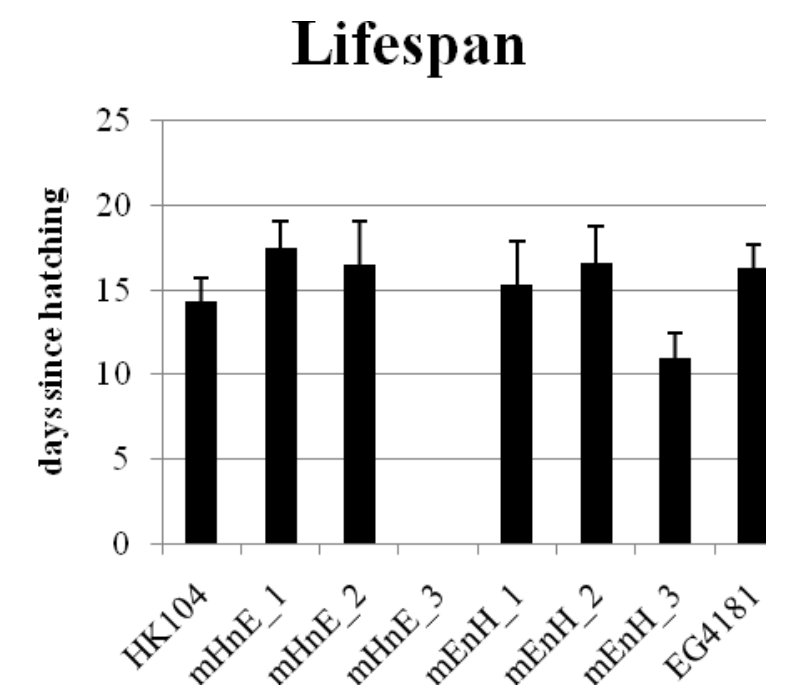

Figure 12. Lifespan in days since hatching for each parental and introgressed line of natural C. briggsae isolates HK104 (high heteroplasmy) and EG4181 (low heteroplasmy). Error bars indicate one standard error. (Data missing for mHnE_3 - see text.) 
Lifespan of the parental and reciprocal hybrid strains from crossing C. briggsae natural isolates PB800 and HK105 are shown in Figure 13. Lifespan of natural $C$. briggsae isolate PB800 (low heteroplasmy) is significantly greater than for HK105 (high heteroplasmy) $(\mathrm{t}(49)=-4.29, \mathrm{p}<0.001$; Table 3$)$. However, lifespan for $\mathrm{mHnP}$ hybrids did not vary significantly among replicates $(\mathrm{F}(2,26)=0.72, \mathrm{p}=0.50)$ nor when pooled and compared to the maternal source isolate HK105 $(\mathrm{t}(55)=-0.54, \mathrm{p}=0.59$; Table 6).

Reciprocal introgression replicates mPnH_1, 2 and 3 also did not differ significantly in total lifespan in days since hatching $(\mathrm{F}(2,27)=2.12, \mathrm{p}=0.14)$, but did exhibit significantly decreased mean lifespan when pooled and compared to the maternal source, PB800 $(\mathrm{t}(51)=-4.73, \mathrm{p}<0.001 ;$ Table 7$)$.

\section{Lifespan}

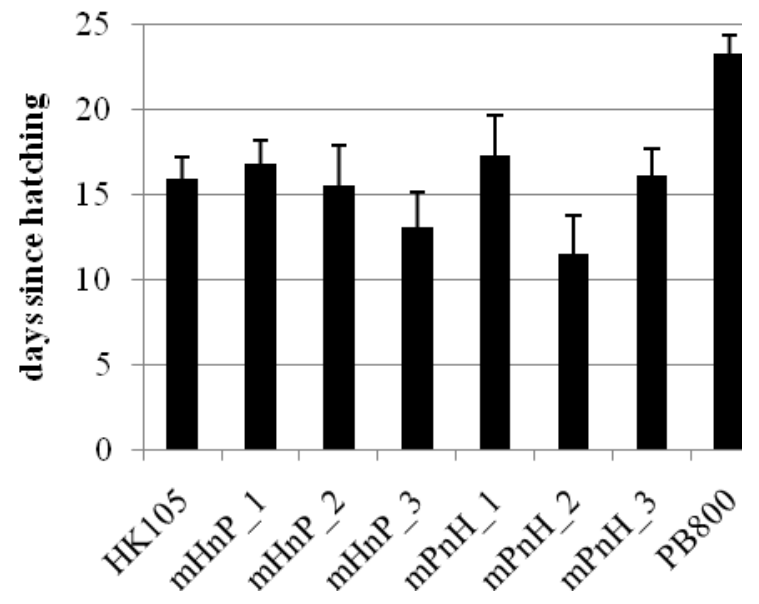

Figure 13. Lifespan in days since hatching for each parental and introgressed line of natural C. briggsae isolates HK105 (high heteroplasmy) and PB800 (low heteroplasmy). Error bars indicate one standard error. 
Pharyngeal pumping

Adult pharyngeal pumping rates of the parental and reciprocal hybrid strains from crossing C. briggsae natural isolates EG4181 and HK104 are shown in Figure 14. As previously determined, low heteroplasmy $C$. briggae natural isolate EG4181 exhibits significantly greater pharyngeal pumping rates every other day through day 10 of adulthood, when compared to the high heteroplasmy isolate HK104 (Table 3) as well as a significantly increased rate of decline in pharyngeal pumping between days 2 and 12 of adulthood $(\mathrm{t}(22)=-2.45, \mathrm{p}<0.05$; Table 3$)$. $C$ briggsae hybrids $\mathrm{mHnE}$ replicates did not

\section{Pharyngeal Pumping}

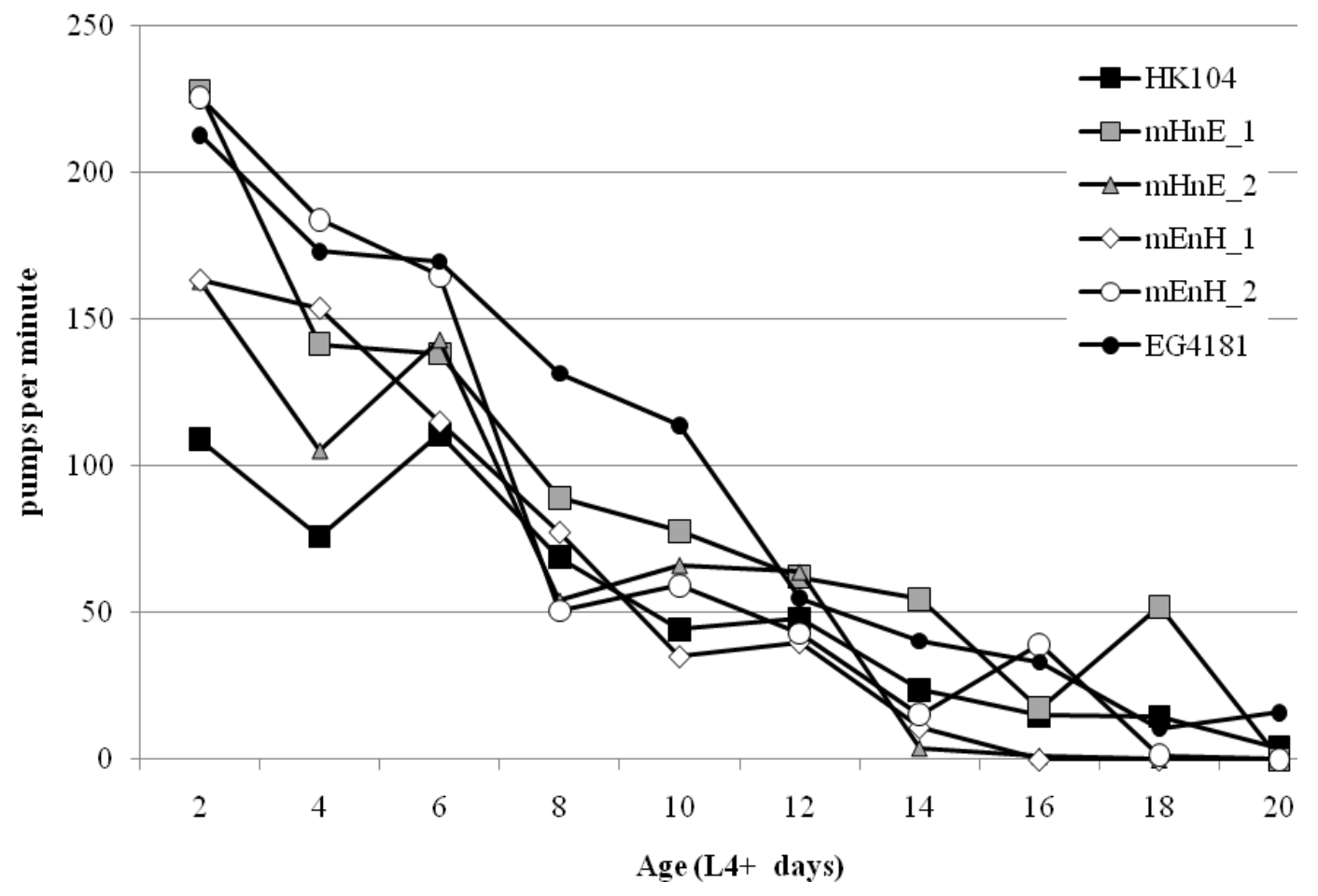

Figure 14. Adult pharyngeal pumping for each parental and two introgressed line of each reciprocal cross of natural C. briggsae isolates HK104 (high heteroplasmy) and EG4181 (low heteroplasmy). 
differ significantly in adult pharyngeal pumping rates nor decline in this rate and displayed increased rates when pooled and compared to maternal source population HK104 on only days 2 and 4 of adulthood (Table 4). C. briggsae mEnH hybrid replicates 1 and 2 differed significantly for only one day of adult pharyngeal pumping and for only two days when pooled and compared to the maternal source, EG4181 (Table 5). Neither comparison between $\mathrm{mEnH}$ replicates nor between pooled replicates and maternal source EG4181 proved significantly different for the rate of decline in pharyngeal pumping between 2 and 12 days of adulthood (Table 5).

\section{Pharyngeal Pumping}

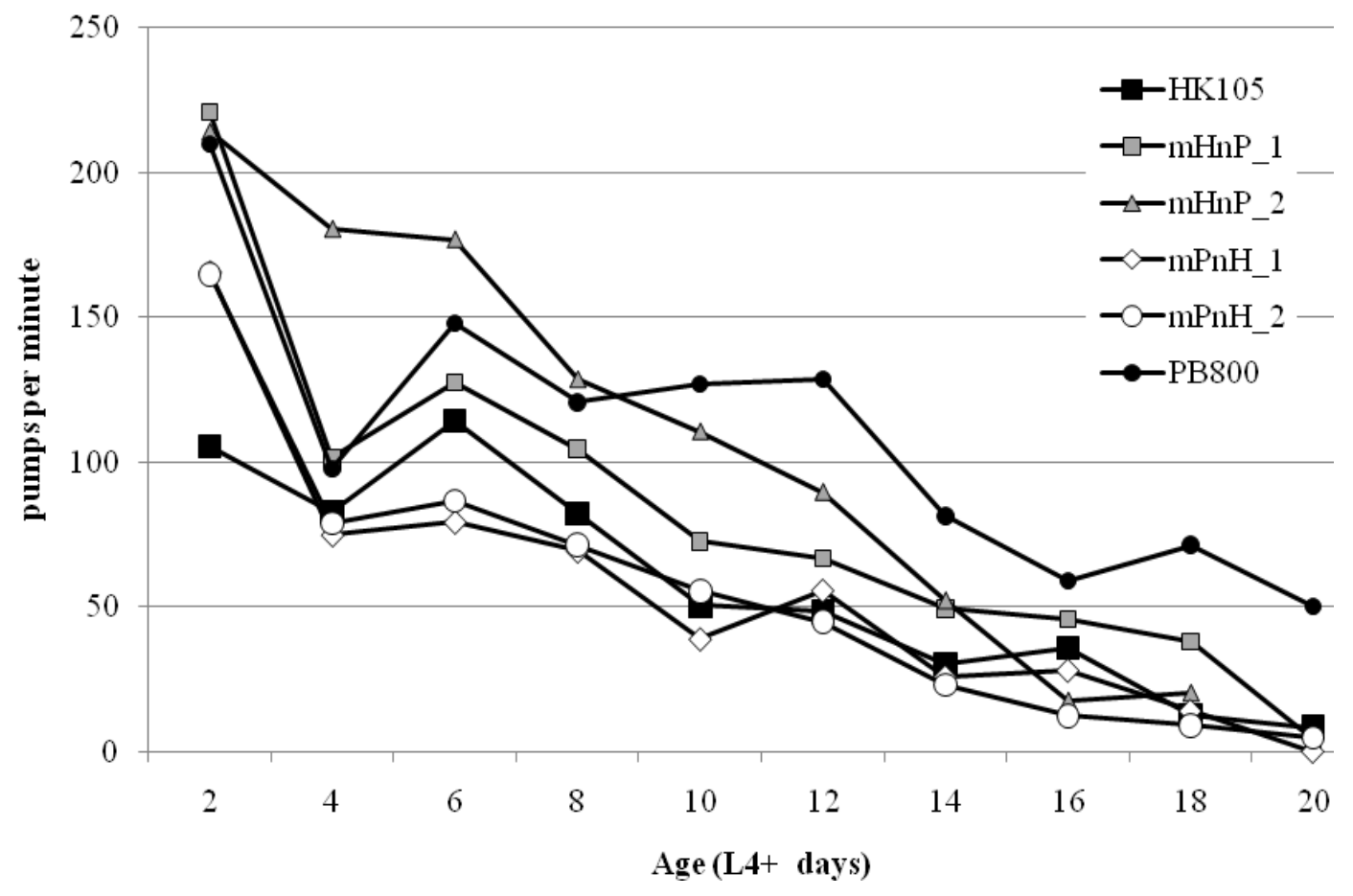

Figure 15. Adult pharyngeal pumping for each parental and two introgressed line of each reciprocal cross of natural C. briggsae isolates HK105 (high heteroplasmy) and PB800 (low heteroplasmy). 
Adult pharyngeal pumping rates of the parental and reciprocal hybrid strains from crossing C. briggsae natural isolates PB800 and HK105 are shown in Figure 15. Natural C. briggsae isolate PB800 (low heteroplasmy) exhibited significantly increased pharyngeal pumping for most days of adulthood measured compared to HK105 (high heteroplasmy), although the decline in pharyngeal pumping was not significantly different between these two isolates (Table 3). Adult pharyngeal pumping of $C$. briggsae hybrids mHnP was significantly different between replicates on only one day but significantly greater than their maternal source, HK105, on four of ten days measured as was the rate of decline in pharyngeal pumping (Table 6). For $\mathrm{mPnH}$ hybrid $C$. briggsae, pharyngeal pumping rates did not differ significantly between replicates but were significantly decreased for all but the first two days of adulthood when compared to the maternal source isolate, PB800 (Table 7); however, due to the lack of a significant difference between the pooled hybrid pharyngeal pumping and that of PB800 on the first day of measurement (day 2), there was no significant difference in decline of pumping rates (Table 7 ).

\section{Larval Growth and Development}

Length of larvae at four time points during development of the parental and reciprocal hybrid strains from crossing C. briggsae natural isolates EG4181 and HK104 are shown in Figure 16. Only larval length at 14 hours after hatching was significantly greater in natural isolate HK104 compared to EG4181 (t(66)=2.9, p<0.05; Table 3). 
Length of mHnE larvae at the first three of four time points varied significantly among replicates and was significantly greater for all replicates compared to the maternal source isolate, HK104, as was the rate of growth in length between the first and final time point (Table 4). For mEnH hybrids, length at each time point and the change in length overall was significantly different among replicates but at only one time point when compared to the maternal source, EG4181 (Table 5).

\section{Larval Growth}

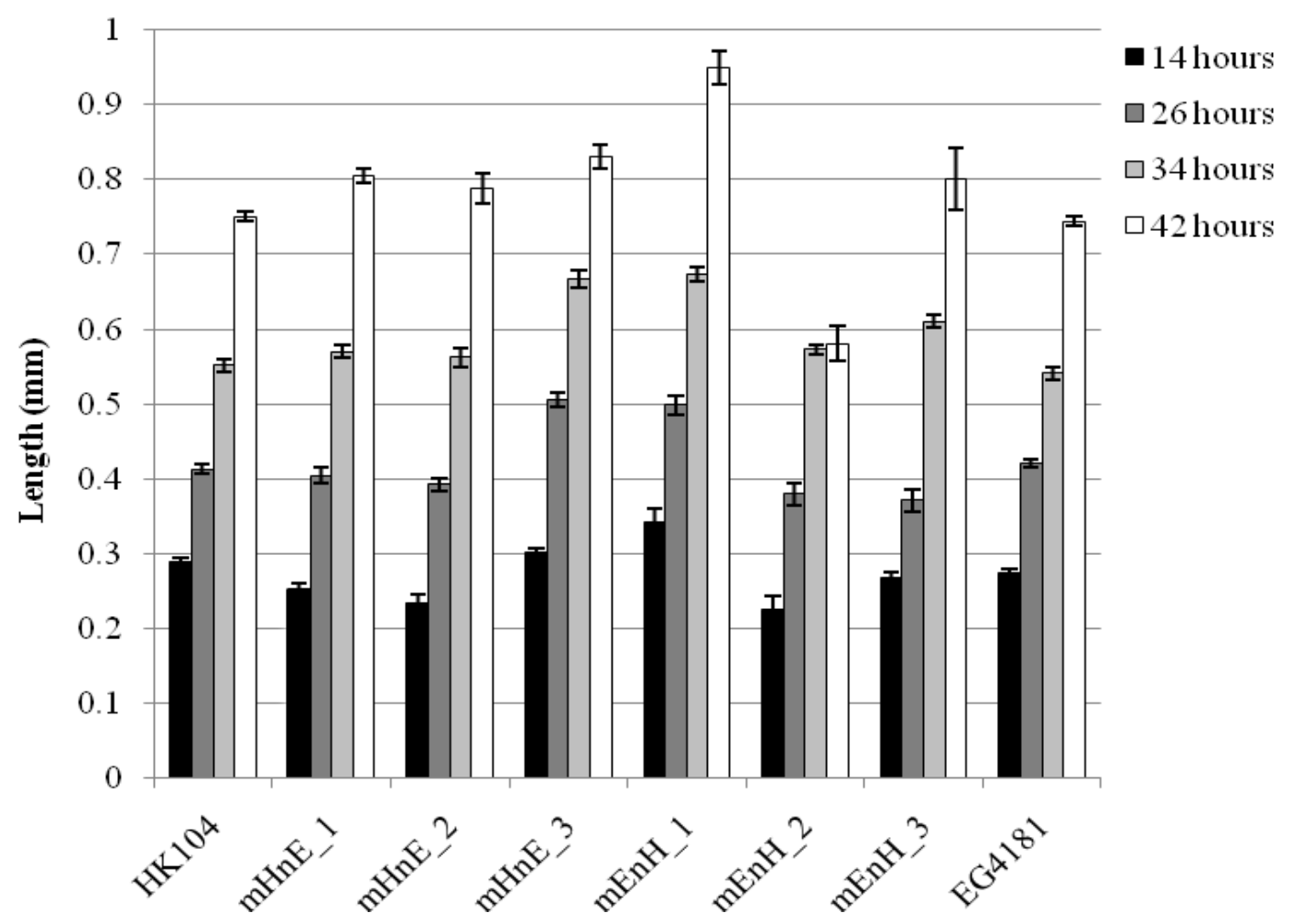

Figure 16. Length of larvae from each parental and introgressed line of natural C. briggsae isolates HK104 (high heteroplasmy) and EG4181 (low heteroplasmy). Bar color indicates length at 14, 26, 34 and 42 hours after hatching, as shown in the figure legend, corresponding to each of four larval stages. Error bars indicate one standard error. 
Length of larvae at four time points during development of the parental and reciprocal hybrid lines from crossing $C$. briggsae natural isolates PB800 and HK105 are shown in Figure 17. Length of $C$. briggsae isolate HK105 larvae was significantly greater than for PB800 at 14 hours after hatching, but significantly less than PB800 at 26, 34 and 42 hours after hatching resulting in an overall increased rate of growth and ultimate size in PB800 larvae compared to HK105 (Table 3). Between mHnP hybrid replicates, larval length varied significantly only at 14 hours after hatching and, accordingly, the rate

\section{Larval Growth}

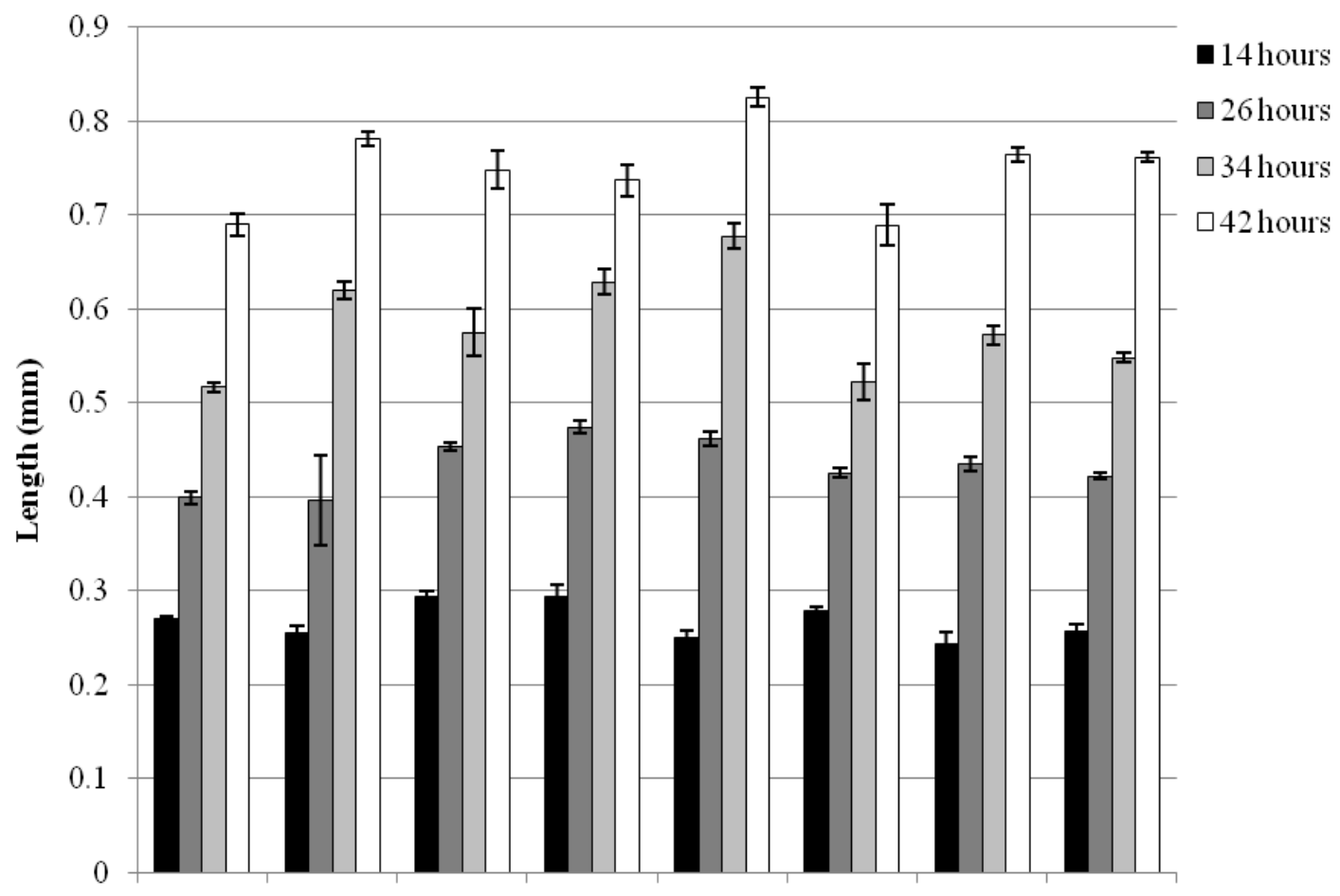

HK105 mHnP $1 \mathrm{mHnP} 2 \mathrm{mHnP} 3 \mathrm{mPnH} 1 \mathrm{mPnH} 2 \mathrm{mPnH} 3 \mathrm{~PB} 800$

Figure 17. Length of larvae from each parental and introgressed line of natural C. briggsae isolates HK105 (high heteroplasmy) and PB800 (low heteroplasmy). Bar color indicates length at 14, 26, 34 and 42 hours after hatching, as shown in the figure legend, corresponding to each of four larval stages. Error bars indicate one standard error. 
of growth in the hybrid larvae between the first and final time point varied significantly among these hybrid replicates (Table 6). When pooled, $\mathrm{mHnP}$ hybrids were longer at the last three time points and grew faster than the natural isolate HK105 (Table 6). $C$.

briggsae hybrid $\mathrm{mPnH}$ larval length varied significantly among replicates for all but the first time point and also differed in rate of growth (Table 7) however did not differ significantly from the maternal source, PB800, except for the two middle measurements (Table 7).

\section{Adult Growth}

Length of adults at three time points of the parental and reciprocal hybrid strains from crossing C. briggsae natural isolates EG4181 and HK104 are shown in Figure 18. Although natural isolate HK104 length on just the first day of adulthood was greater than that of natural isolate EG4181, the latter grew faster over the first twelve days of adulthood (Table 3). Replicates 1 and 2 of hybrid cross mHnE did not differ significantly in length or growth in length over adulthood and were significantly shorter and grew slower than the maternal source of these hybrids, HK104, when pooled (Table 4). Similarly, mEnH hybrid replicates 1 and 2 did not differ in length or growth in length as adults but were significantly longer than their putative mitochondrial source isolate EG4181 on the first day of adulthood resulting in a significantly decreased rate of adult growth when combined and compared to EG4181 (Table 5). 
Length of adults at three time points of the parental and reciprocal hybrid strains from crossing C. briggsae natural isolates PB800 and HK105 are shown in Figure 19. Natural isolate PB800 was significantly longer than HK105 at the start of adulthood, yet neither was significantly larger later in adulthood nor grew faster in length (Table 3). Hybrid $\mathrm{mHnP}$ replicates were similar at the first and final measurements of adult length, but the second replicate was significantly longer than the first on day 6 resulting in an increased rate of growth in length between 0 and 6 days compared to the first replicate (Table 6). When pooled, both $\mathrm{mHnP}$ replicates were significantly longer than their

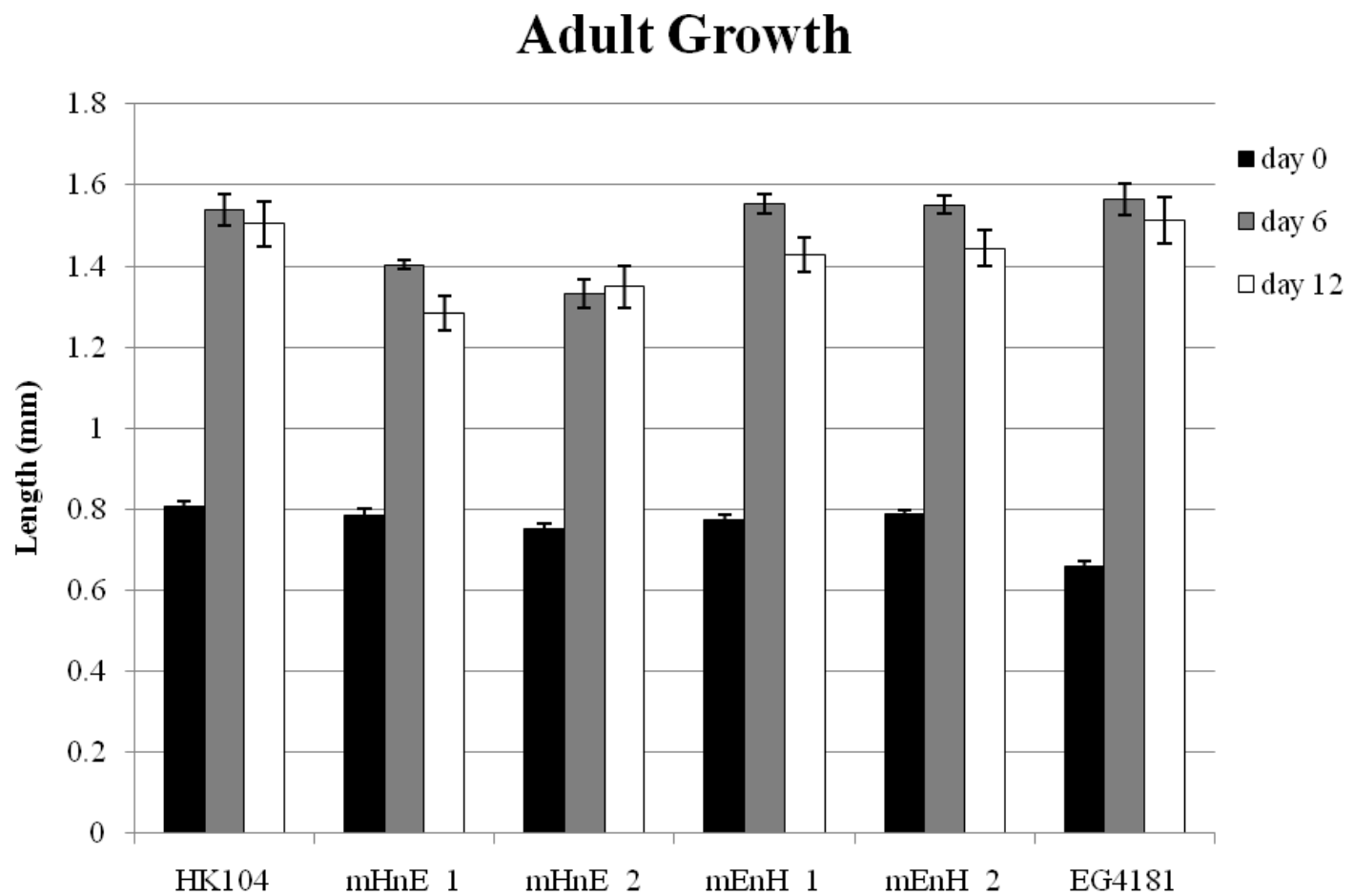

Figure 18. Length of adults from each parental and introgressed line of natural C. briggsae isolates HK104 (high heteroplasmy) and EG4181 (low heteroplasmy). Bar color indicates length at 0, 6, 12 days of maturity, as shown in the figure legend. Error bars indicate one standard error. 
maternal source HK105 on the first day of adult measurements but shorter on the final day of measurements and thus grew slower over this time period than HK105 (Table 6).

No significant difference in adult length and growth exists between replicates of $\mathrm{mPnH}$, yet together were shorter than the maternal source PB800 and so these hybrids grew faster over adulthood compared to PB800 (Table 7).

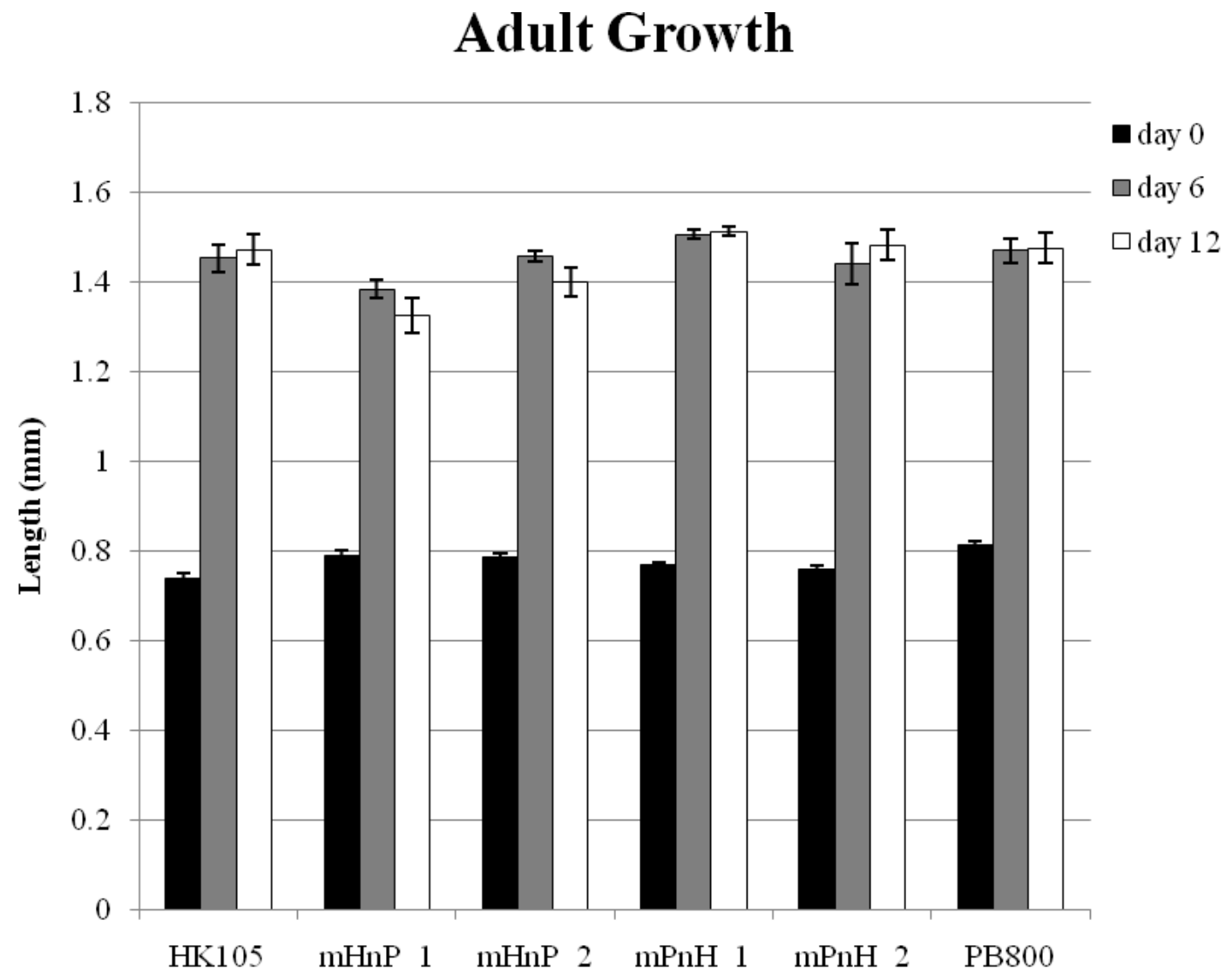

Figure 19. Length of adults from each parental and introgressed line of natural C. briggsae isolates HK105 (high heteroplasmy) and PB800 (low heteroplasmy). Bar color indicates length at 0, 6, 12 days of maturity, as shown in the figure legend. Error bars indicate one standard error. 


\section{Oxygen consumption}

Whole-organism oxygen consumption rates of the parental and reciprocal hybrid strains from crossing C. briggsae natural isolates EG4181 and HK104 are shown in Figure 20. There is no significant difference between rates of young adult oxygen consumption between high heteroplasmy isolate HK104 and low heteroplasmy isolate EG4181 $(\mathrm{t}(2)=0.70, \mathrm{p}=0.56$; Table 3). Two replicates of the hybrid line $\mathrm{mHnE}$ also do not differ in rates of oxygen consumption $(\mathrm{t}(2)=-0.96, \mathrm{p}=0.43)$ but together consume more oxygen per hour per mg protein than the natural isolate HK104 $(\mathrm{t}(4)=-2.51, \mathrm{p}=0.07$;

Table 4). Hybrid mEnH replicates consume oxygen at significantly different rates

\section{Oxygen Consumption}

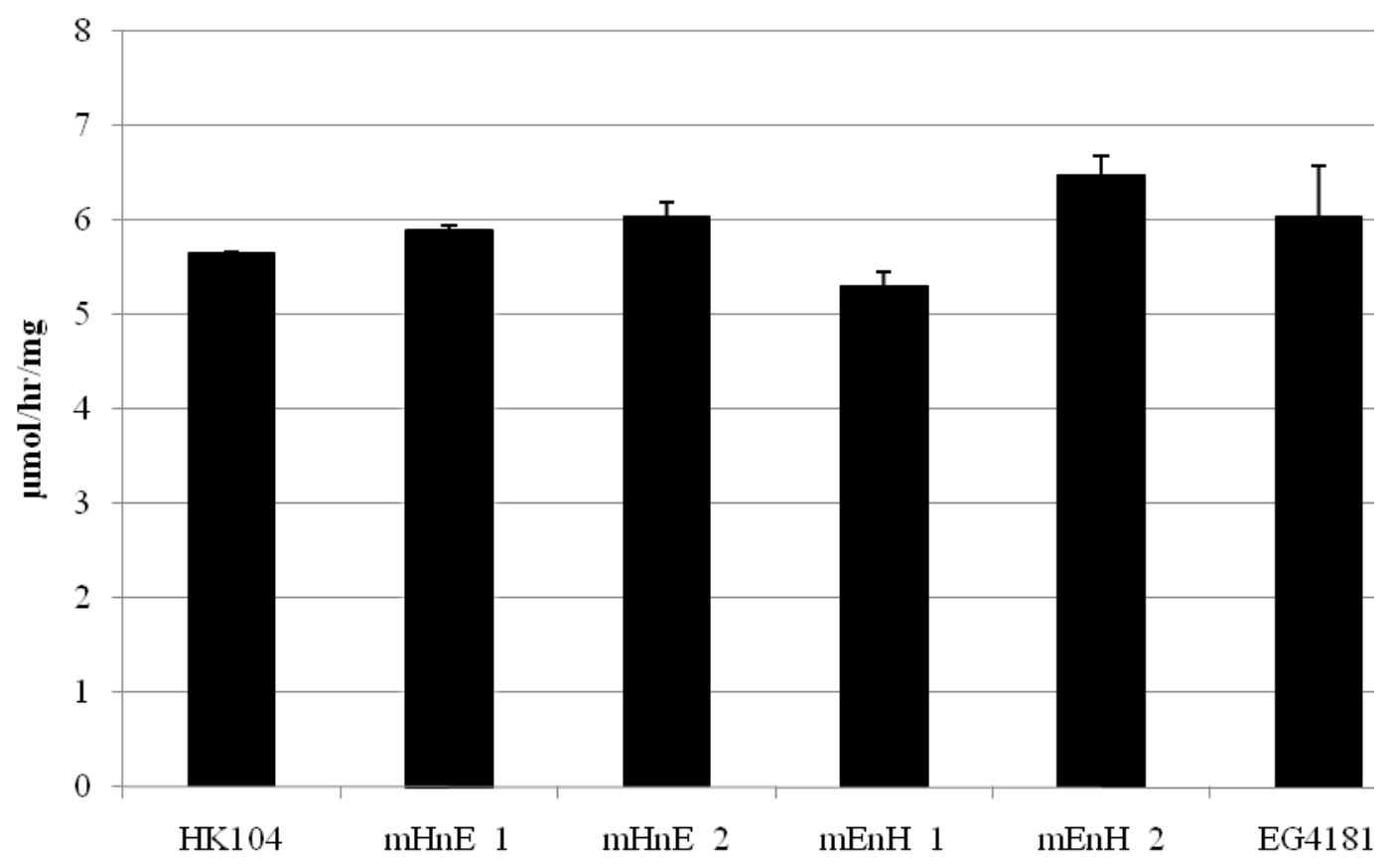

Figure 20. Whole-organism oxygen consumption of young adults for each parental and introgressed line of natural C. briggsae isolates HK104 (high heteroplasmy) and EG4181 (low heteroplasmy). Error bars indicate one standard error. 
$(\mathrm{t}(2)=-4.80, \mathrm{p}=0.04)$ but not when pooled and compared to the maternal source natural isolate EG4181 $(\mathrm{t}(4)=0.23, \mathrm{p}=0.82$; Table 5).

Whole organism oxygen consumption rates of the parental and reciprocal hybrid strains from crossing $C$. briggsae natural isolates PB800 and HK105 are shown in Figure 21. The natural isolates PB800 and HK105 do not consume oxygen at significantly different rates $(\mathrm{t}(2)=-0.96, \mathrm{p}=0.44$; Table 3$)$, despite significant differences in heteroplasmy between them. Rates of oxygen consumption vary significantly between three replicates of $\mathrm{mHnP}$ hybrids $(\mathrm{F}(2,3)=29.39, \mathrm{p}=0.01)$ but not when combined and compared to the putative source of mitochondria from these crosses, HK105 $(\mathrm{t}(6)=0.11$,

\section{Oxygen Consumption}

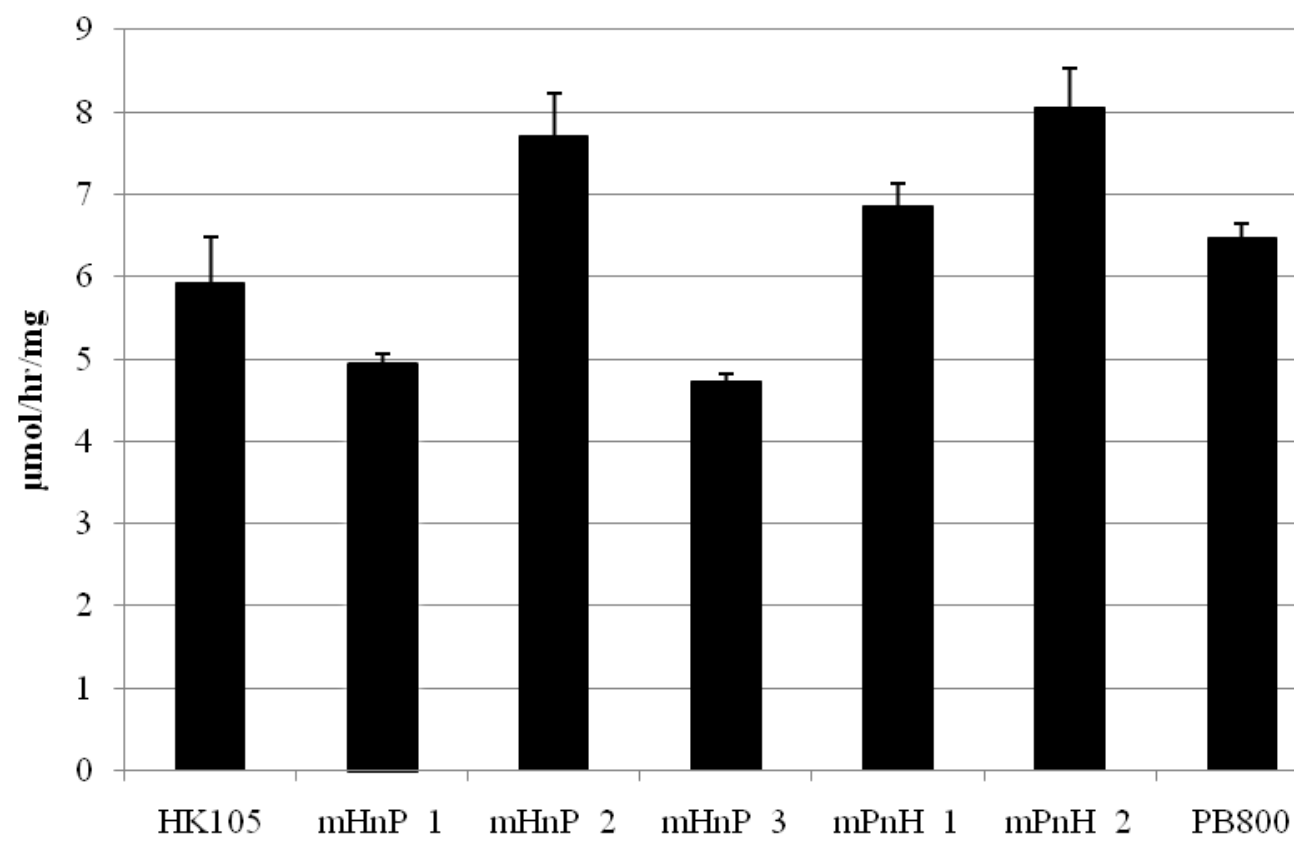

Figure 21. Whole-organism oxygen consumption of young adults for each parental and introgressed line of natural C. briggsae isolates HK105 (high heteroplasmy) and PB800 (low heteroplasmy). Error bars indicate one standard error. 
$\mathrm{p}=0.91$; Table 6). Oxygen consumption of $\mathrm{mPnH}$ hybrids was not significantly different between two replicates, nor between these and the putative source of mitochondria, natural isolate $\mathrm{PB} 800(\mathrm{t}(4)=1.58, \mathrm{p}=0.19$; Table 7). 


\begin{tabular}{lll}
\hline & HK104 vs. EG4181 & HK105 vs. PB800 \\
\cline { 2 - 3 } Life history & & \\
\hline Total reproduction & $\mathrm{t}(58)=-12.19 * * *$ & $\mathrm{t}(48)=-6.42 * * *$ \\
Lifespan (days from hatching) & $\mathrm{t}(43)=-1.62$ & $\mathrm{t}(49)=-4.29 * * *$
\end{tabular}

Pharyngeal pumping (pumps/minute)

\begin{tabular}{lll}
\hline L4+2 days & $\mathrm{t}(26)=-4.60 * * *$ & $\mathrm{t}(25)=-3.75 * * *$ \\
L4+4 days & $\mathrm{t}(26)=-4.60 * * *$ & $\mathrm{t}(26)=-0.75$ \\
L4+6 days & $\mathrm{t}(25)=-2.84 * *$ & $\mathrm{t}(26)=-1.64$ \\
L4+8 days & $\mathrm{t}(24)=-3.41^{* *}$ & $\mathrm{t}(26)=-2.42 *$ \\
L4+10 days & $\mathrm{t}(24)=-3.59 * *$ & $\mathrm{t}(25)=-4.69 * * *$ \\
L4+12 days & $\mathrm{t}(22)=-0.34$ & $\mathrm{t}(25)=-4.67 * * *$ \\
L4+14 days & $\mathrm{t}(17)=-1.00$ & $\mathrm{t}(25)=-2.98 * *$ \\
L4+16 days & $\mathrm{t}(13)=-1.49$ & $\mathrm{t}(24)=-1.27$ \\
L4+18 days & $\mathrm{t}(10)=0.40$ & $\mathrm{t}(23)=-4.54 * * *$ \\
L4+20 days & $\mathrm{t}(7)=-1.14$ & $\mathrm{t}(22)=-3.11 * *$ \\
Rate of decline between 2 and 12 days post-L4 (per day) & $\mathrm{t}(22)=-2.45 *$ & $\mathrm{t}(25)=-0.72$
\end{tabular}

Larval growth (length in mm)

$\begin{array}{lll}14 \text { hours after hatching } & \mathrm{t}(66)=2.09^{*} & \mathrm{t}(38)=2.19^{*} \\ 26 \text { hours after hatching } & \mathrm{t}(56)=-0.82 & \mathrm{t}(50)=-2.96^{* *} \\ 34 \text { hours after hatching } & \mathrm{t}(51)=0.90 & \mathrm{t}(66)=-4.21^{* * *} \\ 42 \text { hours after hatching } & \mathrm{t}(52)=0.78 & \mathrm{t}(50)=-6.27 * * * \\ \text { Rate of growth between 14 and 42 hours after hatching } & \mathrm{t}(52)=-1.56 & \mathrm{t}(35)=-4.80^{* * *}\end{array}$
(per hour)

Adult growth (length in mm)

$\begin{array}{lll}\text { Day 0 (L4) } & \mathrm{t}(30)=7.99 * * * & \mathrm{t}(34)=-5.56^{* * *} \\ \text { Day 6 (past L4) } & \mathrm{t}(28)=-0.48 & \mathrm{t}(27)=-0.43 \\ \text { Day 12 (past L4) } & \mathrm{t}(22)=-0.10 & \mathrm{t}(26)=-0.07 \\ \text { Rate of growth between days 0 and 6 (per day) } & \mathrm{t}(28)=-3.73^{* * *} & \mathrm{t}(27)=1.63 \\ \text { Rate of growth between days 0 and 12 (per day) } & \mathrm{t}(22)=-2.08^{*} & \mathrm{t}(26)=1.66 \\ & & \\ \text { Oxygen consumption } & \mathrm{t}(2)=0.70 & \mathrm{t}(2)=-0.96\end{array}$

Table 3. Test statistics per trait from comparisons between pairs of natural isolate $C$. briggsae progenitors used for hybridization. High ND5 deletion heteroplasmy isolates are HK104 and HK105; low ND5 deletion heteroplasmy isolates are EG4181 and PB800. (*,**, and *** denote significance at $\alpha=0.05,0.01$, and 0.001 , respectively.) 


\section{mHK104 x nEG4181}

hybrid replicates

pooled hybrids vs. maternal parent

Life history

Total reproduction

Lifespan (days from hatching)

$\begin{array}{ll}\mathrm{t}(32)=7.58 * * * & \mathrm{t}(52)=4.19 * * * \\ \mathrm{t}(16)=0.32 & \mathrm{t}(38)=1.20\end{array}$

Pharyngeal pumping (pumps/minute)

\begin{tabular}{lll}
\hline L4+2 days & $\mathrm{t}(24)=1.58$ & $\mathrm{t}(38)=2.50^{*}$ \\
L4+4 days & $\mathrm{t}(20)=1.22$ & $\mathrm{t}(34)=2.06^{*}$ \\
L4+6 days & $\mathrm{t}(17)=-0.28$ & $\mathrm{t}(30)=2.03$ \\
L4+8 days & $\mathrm{t}(17)=1.28$ & $\mathrm{t}(30)=0.19$ \\
L4+10 days & $\mathrm{t}(14)=0.40$ & $\mathrm{t}(27)=1.52$ \\
L4+12 days & $\mathrm{t}(11)=-0.06$ & $\mathrm{t}(23)=0.79$ \\
L4+14 days & $\mathrm{t}(6)=1.53$ & $\mathrm{t}(17)=-0.03$ \\
L4+16 days & $\mathrm{t}(4)=0.92$ & $\mathrm{t}(12)=-0.50$ \\
L4+18 days & $\mathrm{NA}$ & $\mathrm{t}(7)=0.78$ \\
L4+20 days & $\mathrm{NA}$ & $\mathrm{t}(5)=-0.77$ \\
Rate of decline between 2 and 12 days post-L4 (per day) & $\mathrm{t}(11)=0.30$ & $\mathrm{t}(23)=02.71^{*}$
\end{tabular}

Larval growth (length in mm)

\begin{tabular}{lll}
\hline 14 hours after hatching & $\mathrm{F}(2,24)=19.22 * * *$ & $\mathrm{t}(59)=-2.81 * *$ \\
26 hours after hatching & $\mathrm{F}(2,26)=47.41^{* * *}$ & $\mathrm{t}(52)=2.10 *$ \\
34 hours after hatching & $\mathrm{F}(2,32)=25.56 * * *$ & $\mathrm{t}(58)=3.38 * *$ \\
42 hours after hatching & $\mathrm{F}(2,44)=1.80$ & $\mathrm{t}(72)=4.38 * * *$ \\
Rate of growth between 14 and 42 hours after hatching & $\mathrm{F}(2,24)=0.52$ & $\mathrm{t}(52)=5.37 * * *$
\end{tabular}
(per hour)

Adult growth (length in $\mathbf{m m}$ )

\begin{tabular}{lll}
\hline Day 0 (L4) & $\mathrm{t}(18)=1.76$ & $\mathrm{t}(45)=-2.64 *$ \\
Day 6 (past L4) & $\mathrm{t}(18)=1.95$ & $\mathrm{t}(34)=-4.23^{* * *}$ \\
Day 12 (past L4) & $\mathrm{t}(11)=-0.99$ & $\mathrm{t}(25)=-2.89^{*}$ \\
Rate of growth between days 0 and 6 (per day) & $\mathrm{t}(18)=1.03$ & $\mathrm{t}(34)=-4.05^{* * *}$ \\
Rate of growth between days 0 and 12 (per day) & $\mathrm{t}(11)=-1.70$ & $\mathrm{t}(25)=-2.40^{*}$
\end{tabular}

Oxygen consumption

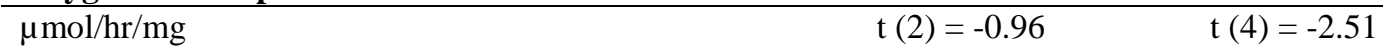

Table 4. Test statistics per trait from comparisons between or among replicates of mHK104 x nEG4181 hybridization ('hybrid replicates'), and between the replicates pooled and the maternal isolate, HK104. $(*, * *$, and $* * *$ denote significance at $\alpha=0.05,0.01$, and 0.001 , respectively. NA denotes impossible analysis due to small sample sizes.) 


\begin{tabular}{|c|c|c|}
\hline mEG4181 x nHK104 & hybrid replicates & $\begin{array}{l}\text { pooled hybrids vs. } \\
\text { maternal parent }\end{array}$ \\
\hline \multicolumn{3}{|l|}{ Life history } \\
\hline Total reproduction & $\mathrm{F}(2,29)=2.12$ & $\mathrm{t}(70)=-6.32 * * *$ \\
\hline Lifespan (days from hatching) & $F(2,26)=2.17$ & $t(47)=-1.62$ \\
\hline \multicolumn{3}{|l|}{ Pharyngeal pumping (pumps/minute) } \\
\hline $\mathrm{L} 4+2$ days & $\mathrm{t}(17)=-2.09$ & $\mathrm{t}(31)=-0.91$ \\
\hline L4+4 days & $\mathrm{t}(17)=-1.19$ & $\mathrm{t}(31)=-0.25$ \\
\hline L4+6 days & $t(16)=-2.53^{*}$ & $\mathrm{t}(30)=-1.71$ \\
\hline L4+8 days & $\mathrm{t}(14)=1.16$ & $\mathrm{t}(27)=-3.60 * *$ \\
\hline L4+10 days & $\mathrm{t}(14)=-1.43$ & $\mathrm{t}(27)=-4.22 * * *$ \\
\hline L4+12 days & $\mathrm{t}(12)=-0.13$ & $t(24)=-0.75$ \\
\hline L4+14 days & $t(8)=-0.36$ & $t(16)=-1.84$ \\
\hline L4+16 days & $\mathrm{t}(5)=-1.00$ & $\mathrm{t}(12)=-0.26$ \\
\hline L4+18 days & $\mathrm{t}(3)=-0.77$ & $\mathrm{t}(9)=-1.48$ \\
\hline L4+20 days & NA & $t(6)=-1.41$ \\
\hline Rate of decline between 2 and 12 days post-L4 (per day) & $\mathrm{t}(12)=-1.71$ & $\mathrm{t}(24)=-0.20$ \\
\hline \multicolumn{3}{|l|}{ Larval growth (length in mm) } \\
\hline 14 hours after hatching & $\mathrm{F}(2,20)=12.81 * * *$ & $\mathrm{t}(55)=1.59$ \\
\hline 26 hours after hatching & $\mathrm{F}(2,20)=26.16^{* * *}$ & $t(54)=0.14$ \\
\hline 34 hours after hatching & $\mathrm{F}(2,23)=31.44 * * *$ & $\mathrm{t}(52)=6.74 * * *$ \\
\hline 42 hours after hatching & $\mathrm{F}(2,36)=35.90 * * *$ & $t(64)=-1.14$ \\
\hline $\begin{array}{l}\text { Rate of growth between } 14 \text { and } 42 \text { hours after hatching } \\
\text { (per hour) }\end{array}$ & $\mathrm{F}(2,16)=6.311^{* *}$ & $\mathrm{t}(44)=1.10$ \\
\hline
\end{tabular}

\section{Adult growth (length in mm)}

\begin{tabular}{lll}
\hline Day 0 (L4) & $\mathrm{t}(18)=-0.91$ & $\mathrm{t}(33)=9.26^{* * * *}$ \\
Day 6 (past L4) & $\mathrm{t}(16)=0.06$ & $\mathrm{t}(30)=-0.34$ \\
Day 12 (past L4) & $\mathrm{t}(12)=-0.266$ & $\mathrm{t}(22)=-1.26$ \\
Rate of growth between days 0 and 6 (per day) & $\mathrm{t}(16)=0.17$ & $\mathrm{t}(30)=-3.42^{* * *}$ \\
Rate of growth between days 0 and 12 (per day) & $\mathrm{t}(12)=-0.07$ & $\mathrm{t}(22)=-3.09 * *$
\end{tabular}

\section{Oxygen consumption}

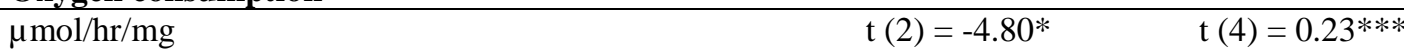

Table 5. Test statistics per trait from comparisons between or among replicates of mEG4181 x nHK104 hybridization ('hybrid replicates'), and between the replicates pooled and the maternal isolate, EG4181. (*, $* *$, and $* * *$ denote significance at $\alpha=0.05,0.01$, and 0.001 , respectively. NA denotes impossible analysis due to small sample sizes.) 


\begin{tabular}{lll}
\hline mHK105 $\mathbf{n P B 8 0}$ & hybrid replicates & $\begin{array}{l}\text { pooled hybrids vs. } \\
\text { maternal parent }\end{array}$ \\
\cline { 2 - 3 } Life history & $\mathrm{F}(2,31)=8.50^{* *}$ & $\mathrm{t}(52)=5.86^{* * *}$ \\
\hline Total reproduction & $\mathrm{F}(2,26)=0.72$ & $\mathrm{t}(55)=-0.54$ \\
Lifespan (days from hatching) & & \\
Pharyngeal pumping (pumps/minute) & $\mathrm{t}(18)=0.26$ & $\mathrm{t}(33)=5.27^{* * *}$ \\
\hline L4+2 days & $\mathrm{t}(18)=-2.60 *$ & $\mathrm{t}(33)=2.54^{*}$ \\
L4+4 days & $\mathrm{t}(16)=-2.03$ & $\mathrm{t}(31)=2.18^{*}$ \\
L4+6 days & $\mathrm{t}(16)=-0.80$ & $\mathrm{t}(31)=2.01$ \\
L4+8 days & $\mathrm{t}(16)=-1.34$ & $\mathrm{t}(31)=2.40^{*}$ \\
L4+10 days & $\mathrm{t}(14)=-0.68$ & $\mathrm{t}(29)=1.53$ \\
L4+12 days & $\mathrm{t}(8)=-0.07$ & $\mathrm{t}(23)=1.12$ \\
L4+14 days & $\mathrm{t}(6)=0.74$ & $\mathrm{t}(20)=-0.04$ \\
L4+16 days & $\mathrm{t}(1)=1.73$ & $\mathrm{t}(15)=1.73$ \\
L4+18 days & $\mathrm{NA}$ & $\mathrm{t}(13)=-0.38$ \\
L4+20 days & $\mathrm{t}(14)=0.48$ & $\mathrm{t}(29)=2.57^{*}$ \\
Rate of decline between 2 and 12 days post-L4 (per &
\end{tabular}
day)

Larval growth (length in mm)

\begin{tabular}{lll}
\hline 14 hours after hatching & $\mathrm{F}(2,20)=8.27 * *$ & $\mathrm{t}(45)=1.26$ \\
26 hours after hatching & $\mathrm{F}(2,23)=1.97$ & $\mathrm{t}(51)=2.25 *$ \\
34 hours after hatching & $\mathrm{F}(2,234)=2.30$ & $\mathrm{t}(59)=7.22 * * *$ \\
42 hours after hatching & $\mathrm{F}(2,35)=2.09$ & $\mathrm{t}(57)=4.46^{* * *}$ \\
Rate of growth between 14 and 42 hours after hatching & $\mathrm{F}(2,20)=4.45^{*}$ & $\mathrm{t}(42)=3.76^{* * *}$
\end{tabular}
(per hour)

Adult growth (length in mm)

\begin{tabular}{|c|c|c|}
\hline Day 0 (L4) & $\mathrm{t}(18)=0.33$ & $\mathrm{t}(35)=3.77 * * *$ \\
\hline Day 6 (past L4) & $\mathrm{t}(17)=-3.23 * *$ & $\mathrm{t}(32)=-0.97$ \\
\hline Day 12 (past L4) & $\mathrm{t}(14)=-1.51$ & $\mathrm{t}(29)=-2.55^{*}$ \\
\hline $\begin{array}{l}\text { Rate of growth between days } 0 \text { and } 6 \text { (per day) } \\
\text { Rate of growth between days } 0 \text { and } 12 \text { (per day) }\end{array}$ & $\begin{array}{l}\mathrm{t}(17)=-2.60 * \\
\mathrm{t}(14)=-1.35\end{array}$ & $\begin{array}{l}\mathrm{t}(32)=-2.60 * \\
\mathrm{t}(29)=-3.75^{*} * *\end{array}$ \\
\hline \multicolumn{3}{|l|}{ Oxygen consumption } \\
\hline$\mu \mathrm{mol} / \mathrm{hr} / \mathrm{mg}$ & $F(2,3)=29.39 *$ & $t(6)=0.11$ \\
\hline
\end{tabular}

Table 6. Test statistics per trait from comparisons between or among replicates of mHK105 x nPB800 hybridization ('hybrid replicates'), and between the replicates pooled and the maternal isolate, HK105. (*, $* *$, and $* * *$ denote significance at $\alpha=0.05,0.01$, and 0.001 , respectively. NA denotes impossible analysis due to small sample sizes.) 


\begin{tabular}{|c|c|c|}
\hline mPB800 x nHK105 & hybrid replicates & $\begin{array}{l}\text { pooled hybrids vs. } \\
\text { maternal parent }\end{array}$ \\
\hline \multicolumn{3}{|l|}{ Life history } \\
\hline Total reproduction & $\mathrm{F}(2,41)=1.79$ & $\mathrm{t}(72)=-4.93 * * *$ \\
\hline Lifespan (days from hatching) & $F(2,27)=2.12$ & $\mathrm{t}(51)=-4.73 * * *$ \\
\hline \multicolumn{3}{|l|}{ Pharyngeal pumping (pumps/minute) } \\
\hline L4+2 days & $\mathrm{t}(26)=0.02$ & $t(38)=-1.66$ \\
\hline L4+4 days & $t(25)=-0.18$ & $\mathrm{t}(38)=-1.10$ \\
\hline L4+6 days & $\mathrm{t}(25)=-0.42$ & $\mathrm{t}(38)=-3.90 * * *$ \\
\hline L4+8 days & $\mathrm{t}(24)=-0.12$ & $\mathrm{t}(37)=-3.31 * *$ \\
\hline L4+10 days & $\mathrm{t}(22)=-0.98$ & $\mathrm{t}(34)=-5.12 * * *$ \\
\hline L4+12 days & $\mathrm{t}(24)=0.70$ & $\mathrm{t}(36)=-5.29 * * *$ \\
\hline L4+14 days & $\mathrm{t}(21)=0.29$ & $\mathrm{t}(33)=-4.42 * * *$ \\
\hline L4+16 days & $\mathrm{t}(17)=1.55$ & $\mathrm{t}(29)=-2.81 * *$ \\
\hline L4+18 days & $t(16)=0.62$ & $\mathrm{t}(27)=-5.36^{* * *}$ \\
\hline L4+20 days & $t(7)=-0.88$ & $\mathrm{t}(18)=-3.06^{* *}$ \\
\hline Rate of decline between 2 and 12 days post-L4 & $\mathrm{t}(24)=-0.46$ & $\mathrm{t}(35)=0.69$ \\
\hline
\end{tabular}
(per day)

\section{Larval growth (length in mm)}

\begin{tabular}{lll}
\hline 14 hours after hatching & $\mathrm{F}(2,19)=2.63$ & $\mathrm{t}(36)=-0.01$ \\
26 hours after hatching & $\mathrm{F}(2,25)=7.38^{* *}$ & $\mathrm{t}(51)=3.31^{* *}$ \\
34 hours after hatching & $\mathrm{F}(2,25)=31.51^{* * *}$ & $\mathrm{t}(60)=3.12^{* *}$ \\
42 hours after hatching & $\mathrm{F}(2,31)=27.53 * * *$ & $\mathrm{t}(63)=0.86$ \\
Rate of growth between 14 and 42 hours after & $\mathrm{F}(2,18)=22.72^{* * * *}$ & $\mathrm{t}(35)=0.13$
\end{tabular}
hatching (per hour)

Adult growth (length in mm)

\begin{tabular}{lll}
\hline Day 0 (L4) & $\mathrm{t}(28)=0.60$ & $\mathrm{t}(47)=-4.80^{* * * *}$ \\
Day 6 (past L4) & $\mathrm{t}(25)=1.07$ & $\mathrm{t}(39)=-0.10$ \\
Day 12 (past L4) & $\mathrm{t}(24)=0.73$ & $\mathrm{t}(37)=0.52$ \\
Rate of growth between days 0 and 6 (per day) & $\mathrm{t}(24)=0.66$ & $\mathrm{t}(39)=1.10$ \\
Rate of growth between days 0 and 12 (per day) & $\mathrm{t}(24)=0.65$ & $\mathrm{t}(37)=2.05^{*}$ \\
& & \\
Oxygen consumption & $\mathrm{t}(2)=-2.23$ & $\mathrm{t}(4)=1.58$ \\
\hline$\mu \mathrm{mol} / \mathrm{hr} / \mathrm{mg}$ &
\end{tabular}

Table 7. Test statistics per trait from comparisons between or among replicates of mPB800 x nHK105 hybridization ('hybrid replicates'), and between the replicates pooled and the maternal isolate, PB800. (*, $* *$, and $* * *$ denote significance at $\alpha=0.05,0.01$, and 0.001 , respectively.) 


\section{Genotyping}

Qualitative assessment of banding for PCR products containing the $\sim 700 \mathrm{bp}$ partial deletion of ND5 in C. briggsae hybrids and natural isolates used for hybridization revealed unexpected inheritance of the shorter mtDNA sequence. In fact, for each replicate of each introgression and its reciprocal (with one exception), banding patterns were the opposite from those expected given stable inheritance of heteroplasmy levels through the maternal lineage (Table 8). These same results were observed over two separate PCR procedures. To further verify whether mtDNA had been inherited in $C$. briggsae hybrids as expected, quantitative PCR for microsatellites in the mtDNA cytochrome oxidase II (COII) gene was performed. Again, unexpectedly, results from these assays indicated that mitochondria had been inherited through the paternal lineage of most replicate hybrids lines except for those of the mPnH introgressions (Table 8). Subsequent sequencing of the deletion-sized band from only one replicate of these $\mathrm{mPnH}$ hybrids revealed that a deletion of novel size had accumulated in the mtDNA of this line. 


\begin{tabular}{|c|c|c|c|c|c|c|}
\hline Mt Origin & $\begin{array}{c}\text { Nuclear } \\
\text { Origin } \\
\end{array}$ & Rep & Annotation & $\begin{array}{c}\text { Expected ND5 } \\
\text { deletion \% } \\
\end{array}$ & $\begin{array}{c}\text { Actual } N D 5 \\
\text { deletion } \% \\
\end{array}$ & $\begin{array}{c}\text { Mt } \\
\text { Genotype } \\
\end{array}$ \\
\hline \multirow{3}{*}{ HK104 } & & 1 & mHnE_1 & \multirow{3}{*}{$\sim 50$} & $\sim 50$ & EG4181 \\
\hline & EG4181 & 2 & mHnE_2 & & $\sim 0$ & HK104 \\
\hline & & 3 & mHnE_3 & & $\sim 0$ & EG4181 \\
\hline \multirow{3}{*}{ EG4181 } & & 1 & mEnH_1 & \multirow{3}{*}{$\sim 0$} & $\sim 50$ & HK104 \\
\hline & HK104 & 2 & mEnH_2 & & $\sim 50$ & HK104 \\
\hline & & 3 & mEnH_3 & & $\sim 50$ & HK104 \\
\hline \multirow{3}{*}{ HK105 } & & 1 & mHnP_1 & \multirow{3}{*}{$\sim 50$} & $\sim 5$ & HK105 \\
\hline & PB800 & 2 & mHnP_2 & & $\sim 5$ & ND \\
\hline & & 3 & mHnP_3 & & $\sim 5$ & PB800 \\
\hline \multirow{3}{*}{ PB800 } & & 1 & mPnH_1 & \multirow{3}{*}{$\sim 5$} & $\sim 50 * *$ & PB800* \\
\hline & HK105 & 2 & mPnH_2 & & $\sim 50 * *$ & PB800* \\
\hline & & 3 & mPnH_3 & & $\sim 50 * *$ & PB800* \\
\hline
\end{tabular}

Table 8. Expectations of heteroplasmy levels and outcomes from crossing $C$. briggsae natural isolates with high and low ND5 deletion levels. Actual ND5 deletion \% are based on qualitative analyses of PCR and gel electrophoresis for the region of mtDNA missing when the ND5 deletion is present. Mt genotypes were determined by isolate-specific SNPs in the COII gene. (ND=not determined.) *Inheritance of mitochondria is maternal in all replicates of this hybridization only. **While the banding pattern of ND5 deletion region for all replicates of this cross mimic that for the paternal population, thus contradicting results of mtDNA genotyping, sequencing of the deleted region from one of these replicates indicates a deletion of novel size and location. 


\section{DISCUSSION}

To establish whether natural mtDNA variation controls the phenotypic variance among isolates from natural populations of the nematode $C$. briggsae, nematodes hybridized from various pairs of natural $C$. briggsae isolates have been analyzed for morphological and physiological traits and compared to respective parental trait means. Assuming that mitochondria are inherited through the maternal lineage, ND5 deletion frequency is transmitted to offspring at a stable frequency, and that epistasis between genomes does not contribute to the traits measured here, the hybrid C. briggsae phenotypes were expected to closely match those of the maternal progenitor natural isolate.

Surprisingly, inheritance of mitochondria through the paternal lineage occurred in half of $C$. briggsae hybrid introgressions. However, paternal transmission of mitochondria is not unheard of; in fact, mitochondria from sperm enter the ovum upon fertilization in most species (Cummins, 2000). Prior to departure from the male gonad, mitochondria in sperm are ubiquinated and then destroyed post-fertilization (Sutovsky, 2003). Although there are a few natural instances of paternally-inherited mitochondria, the persistence of paternal mitochondria occurs more often in interspecific hybrids (Cummins, 2000). In such a case, whether paternal leakage of mitochondria is due to recombination between haplotypes or to evasion of the selective forces that usually eliminate paternal mitochondria remains to be determined (Cummins, 2000). 
Comparison between $C$. briggsae hybrids in which mitochondria were inherited maternally ( $\mathrm{mPnH}$, all replicates) and the maternal progenitor (natural isolate PB800) reveals that intergenomic epistasis contributes to the phenotypic expression of most traits measured here, i.e. reproduction, longevity, and aging. Typically, F1 hybrids experience 'hybrid vigor' denoted by increased fitness relative to parental means. Also known as heterosis, this phenomenon is likely due to overdominance (intralocus interactions that increase fitness), positive epistasis (between parental alleles), or the recovery from inbreeding depression (Lynch, 1991). Most examinations of interlocus interactions provide evidence for the later mechanism to explain hybrid vigor (Schierup and Christiansen, 1996) which was first proposed by Crow (1948) who suggested that such an increase in hybrid fitness is due to the masking of recessive deleterious alleles that may accumulate in isolated populations due to drift. In F2 hybrids and later generations, outbreeding depression can occur upon recombining recessive deleterious alleles of the same parental type and the further break up of positively epistasic alleles which may have coevolved in one or both parental types thus resulting in decreased hybrid fitness (Edmands, 1999).

Reproductive isolation can occur between allopatric populations due to differences in mutation, adaptation to the local environment, and random changes in allele frequencies. Across taxa, most work aimed at elucidating the genetic underpinnings of intrinsic postreproductive isolates has yielded evidence supporting a primary role of epistasis (Coyne and Orr, 2004). While the reduced fitness of hybrid individuals may be 
attributed to epistasis between parental alleles at nuclear loci, the same phenomenon can occur between genomes (cytonuclear interactions) and, lately, much interest has been generated around this concept due to the important role of organellar genes in fitness of both plants and animals (Wolf, 2009). Since gene interactions that increase fitness can generate linkage disequilibrium resulting in the biased inheritance of those genes, the fixation of alleles that maximize fitness at such loci may result in the evolution of coadapted gene complexes (Rand et al., 2004).

To date, much evidence has been accumulated to support the notion that intergenomic coadapted gene complexes play a role in the breakdown of fitness in hybrids between allopatric populations of Tigriopus californicus, an intertidal marine copepod. In fact, second generation hybrids vary from parents in a number of fitness related traits (Burton, 1990; Edmands, 1999), and particular introgressions result in reduced mitochondrial function (Edmands and Burton, 1999; Harrison and Burton, 2005) that correlates to fitness (Willet and Burton, 2001) and is recovered upon restoration of parental genome pairs (Ellison and Burton, 2008). However, the extent to which such cytonuclear epistasis promotes intrinsic postzygotic isolation across taxa has yet to be determined. Perhaps divergence may result from the evolution of cytonuclear coadapted gene complexes more often for organisms with more disparate rates of mutation between genomes, as in T. californicus (Willet and Burton, 2004; Burton et al., 2006; Burton et al., 2007) and C. briggsae (Baer et al., 2007; Phillips et al., 2009; Howe et al., 2010). 
One caveat to the statements made here regarding intergenomic epistasis in $C$. briggsae is the accumulation of mtDNA deletions of novel size in those hybrid lines which inherited mtDNA as expected (Table 8). Since the extent of newly arisen deletions in $\mathrm{mPnH}$ hybrids has not been defined, no speculation can be made as to whether these novel mutations may influence the variation seen among hybrid replicates, and between these hybrids and their maternal progenitor. Interestingly, these novel mutations arose in the mtDNA of a natural isolate identified as producing low levels of in vivo superoxide (Estes et al., in review). However, the mtDNA of this same isolate (PB800) was found to accumulate numerous large heteroplasmic deletions in a mutation-accumulation experiment (Howe et al., 2010) compared to one such event in C. elegans mutationaccumulation experiments (Denver et al., 2000). 


\section{CONCLUSION}

Given the importance of the mitochondrial electron transport chain (ETC) to energy production in most eukaryotes, it is no surprise that mutations affecting electron transport function result in various human disorders (Wallace, 2005) and affect aging in other organisms (Balaben et al., 2005). As for other biological processes, the nematode Caenorhabditis elegans has proved a useful model for understanding the genetic underpinnings of lifespan determination (Hertweck, 2005). Mutations of nuclear or mitochondrial genes that contribute to mitochondrial biogenesis and function can impact longevity, fecundity, and aging in C. elegans (Lee et al., 2003; Rea and Johnson, 2003; Balaben et al., 2005; Rea, 2005; Liau et al., 2007; Rea et al., 2007). However, very little is understood about the direct mechanism(s) through which genetic variation that affects mitochondria controls these traits, and the evolutionary forces that regulate those relationships (Ballard and Whitlock, 2004).

To establish a model system for studying aging and longevity in an evolutionary or population genetics framework, several physiological characteristics have been examined in recently isolated Caenorhabditis briggsae nematodes with naturally occurring mitochondrial genetic variation relevant to the aging process. Previously, superoxide production, reproduction and population growth rates were found to be negatively correlated to heteroplasmy levels of a 700bp deletion present in the mitochondrial genome of most $C$. briggsae isolates (Estes et al., in review). This deletion 
removes around half of an ETC complex I subunit (Howe and Denver, 2008), which is thought to be essential for aerobic ATP production (Janssen et al., 2006). Here, aging and time to reproductive maturity were shown to correlate positively with heteroplasmy among $C$. briggsae isolates. Future work will aim at understanding exactly how this mitochondrial mutation in C. briggsae impacts mitochondrial function.

Experimental hybrid lines of $C$. briggsae were generated from pairs of natural isolates with high and low heteroplasmy levels in an attempt to evaluate the phenotypic effects of a mitochondrial genome from one isolate on the nuclear genetic background of another - which is necessary to show that phenotypic variation among natural isolates is due to mitochondrial genetic variance. Contrary to expectation under the assumption that mitochondria are always inherited maternally, some hybrid F10 carried only paternallyderived mitochondrial genetic information. From C. briggsae hybrids that inherited maternal mitochondria, intergenomic epistasis appears to impact lifespan, fitness, and aging in this species. This latter implication is unsurprising given the close relationship between many gene products from both genomes in mitochondrial function and ATP production. In the future, hybrid $C$. briggsae can be used to examine transmission and maintenance of heteroplasmy levels as well as how drift and natural selection may contribute to observed deletion heteroplasmy levels. 


\section{REFERENCES}

Albertson DG and Thomson JN. 1976. The pharynx of Caenorhabditis elegans. Philosophical Transansactions of the Royal Society London, Series B 275: 299325.

Alexeyev MF, LeDoux SP and Wilson GL. 2004. Mitochondrial DNA and aging. Clinical Science 107: 355-364.

Baer CF, Shaw F, Steding C, Baumgartner M, Hawkins A, Houppert A, Mason N, Reed M, Woodard W, Lynch M, and Anderson WW. 2005. Comparative evolutionary genetics of spontaneous mutations affecting fitness in rhabditid nematodes. Proceedings of the National Academy of Sciences, USA 102(16): 5785-5790.

Baer CF, Miyamoto MM, and Denver DR. 2007. Mutation rate variation in multicellular eukaryotes: causes and consequences. Nature Reviews Genetics 8: 619-631.

Bai Y, Shakeley RM, and Attardi G. 2000. Tight control of respiration by NADH dehydrogenase ND5 subunit gene expression in mouse mitochondria. Molecular and Cellular Biology 20(3): 805-815.

Balaban RS, Nemoto S, and Finkel T. 2005. Mitochondria, oxidants, and aging. Cell 120: 483-495.

Ballard JWO and Whitlock MC. 2004. Incomplete natural history of mitochondria. Molecular Ecology 13: 729-744.

Bandy B and Davison AJ. 1990. Mitochondrial mutations may increase oxidative stress: implications for carcinogenesis and aging? Free Radical Biology and Medicine 8: 523-539.

Beckman KB and Ames BN. 1998. The free radical theory of aging matures. Physiological Reviews 78(2): 547-581.

Bolanowksi MA, Richard LR, Jacobson LA. 1981. Quantitative measures of ageing in the nematode Caenorhabditis elegans. I. Population and longitudinal studies of two behavior parameters. Mechanisms of Ageing and Development 15: 279-295.

Braeckman BP, Houthoofd K, De Vreese A, and Vanfleteren JR. 2002a. Assaying metabolic activity in ageing Caenorhabditis elegans. Mechanisms of Ageing and Development 123: 105-119.

Braeckman BP, Houthoofd K, and Vanfleteren JR. 2002b. Assessing metabolic activity in aging Caenorhabditis elegans: concepts and controversies. Aging Cell 1: 82-88.

Braeckman BP, Houthoofd K, and Vanfleteren JR. 2008. Intermediary metabolism, WormBook, ed. The C. elegans Research Community, WormBook, doi/10.1895/wormbook.1.146.1, http://www.wormbook.org.

Bratic I, Hench J, Henriksson J, Antebi A, Burglin TR, and Trifunovic A. 2009 Mitochondrial DNA level, but not active replicase, is essential for Caenorhabditis elegans development. Nucleic Acids Research 37(6): 1817-1828.

Burton RS. 1990. Hybrid breakdown in developmental time in the copepod Tigriopus californicus. Evolution 44: 1814-1822. 
Burton RS, Ellison CK and Harrison JS. 2006. The sorry state of $F_{2}$ hybrids: Consequences of rapid mitochondrial DNA evolution in allopatric populations. The American Naturalist 168: S14-S24

Burton RS, Byrne RJ, and Rawson PD. 2007. Three divergent mitochondrial genomes from California populations of the copepod Tigriopus californicus. Gene 403: 5359.

Chinnery PF. 2000. The inheritance of mitochondrial DNA heteroplasmy: random drift, selection or both? Trends in Genetics 16(11): 500-505.

Chinnery PF and Turnbull DM. 2000. Mitochondrial DNA mutations in the pathogenesis of human disease. Molecular Medicine Today 6: 425-432.

Chomyn A and Attardi G. 2003. MtDNA mutations in aging and apoptosis. Biochemical and Biophysical Research Communications 304: 519-529.

Collins JJ, Huang C, Hughes S, and Kornfeld K. 2008. The measurement and analysis of age-related changes in Caenorhabditis elegans, WormBook, ed. The C. elegans Research Community, WormBook, doi/10.1895/wormbook.1.137.1, http://www.wormbook.org.

Corral-Debrinski M, Morton T, Lott MT, Shoffner JM, Beal MF, and Wallace DC. 1992. Mitochondrial DNA deletions in human brain: regional variability and increase with advanced age. Nature Genetics 2: 324-329.

Coyne JA and Orr HA. 2004. Speciation. Sinauer Asociates, Sunderland, MA, USA. Croll NA, Smith JM, and Zuckerman BM. 1977. The aging process of the nematode Caenorhabditis elegans in bacterial and axenic culture. Experimental Aging Research 3: 175-189.

Crow JF. 1948. Alternative hypotheses of hybrid vigor. Genetics 33: 477-478.

Cummins JM. 2000. Fertilization and elimination of the paternal mitochondrial genome. Human Reproduction 15(s2): 92-101.

de Grey A. 2005. Reactive oxygen species production in the mitochondrial matrix: implications for the mechanism of mitochondrial mutation accumulation. Rejuvenation Research 8(1): 13-19.

Denver DR, Morris K, Lynch M, Vassilieva LL, and Thomas WK. 2000. High direct estimate of the mutation rate in the mitochondrial genome of Caenorhabditis elegans. Science 289: 2342-2344.

Diaz F, Bayona-Bafaluy MP, Rana M, Mora M, Hao H, and Moreas CT. 2002. Human mitochondrial DNA with large deletions repopulates organelles faster than fulllength genomes under relaxed copy number control. Nucleic Acids Research 30(21): 4626-4633.

Dillin A, Hsu AL, Arantes-Oliveira N, Lehrer_Graiwer J, Hsin H, Fraser AG, Kamath RS, Ahringer J, and Kenyon C. 2002. Rates of behavior and aging specified by mitochondrial function during development. Science 298: 2398-2401.

DiMauro S and Schon EA. 2003. Mitochondria respiratory-chain diseases. New England Journal of Medicine 348: 2656-2668. 
Edmands S. 1999. Heterosis and outbreeding depression in interpopulation crosses spanning a wide range of divergence. Evolution 53:1757-1765.

Edmands S and Burton RS. 1999. Cytochrome $c$ oxidase activity in interpopulation hybrids of a marine copepod: a test for nuclear-nuclear or nuclear-cytoplasmic coadaptation. Evolution 53(6): 1972-1978.

Ellison CK and Burton RS. 2008. Interpopulation hybrid breakdown maps to the mitochondrial genome. Evolution 62(3): 631-638.

Estes S, Coleman-Hulbert AL, Hicks KA, de Haan G, Martha SR, Knapp JB, Smith SW, Stein KC, and Denver DR. In review, BMC Evolutionary Biology.

Ewbank JJ, Barnes TM, Lakowski B, Lussier M, Bussey H, and Hekimi S. 1997. Structural and functional conservation of the Caenorhabditis elegans timing gene clk-1. Science 275: 980-983.

Garigan D, Hsu AL, Fraser AG, Kamath RS, Ahringer J, and Kenyon C. 2002. Genetic analysis of tissue aging in Caenorhabditis elegans: a role for heat-shock factor and bacterial proliferation. Genetics 161: 129-155.

Gems D and Doonan R. 2009. Antioxidant defense and aging in C. elegans. Cell Cycle 8(11): 1681-1687.

Grad LI and Lemire BD. 2004. Mitochondrial complex I mutations in Caenorhabditis elegans produce cytochrome oxidase deficiency, oxidative stress and vitaminresponsive lactic acidosis. Human Molecular Genetics 13(3): 303-314.

Hamilton B, Dong Y, Shindo M, Liu W, Odell I, Ruvkun G, and Lee SS. 2005. A systematic RNAi screen for longevity genes in C. elegans. Genes and Development 19: 1544-1555.

Harman D. 1956. Aging: A theory based on free radical and radiation chemistry. The Journals of Gerontology 11(3): 298-300.

Harrison JS and Burton RS. 2005. Tracing hybrid incompatibilities to single amino acid substitutions. Molecular Biology and Evolution 23(3): 559-564.

Herndon LA, Schmeissner PJ, Dudaronek JM, Brown PA, Listner KM, Sakano Y, Paupard MC, Hall DH, and Driscoll M. 2002. Stochastic and genetic factors influence tissue-specific decline in ageing C. elegans. Nature 419: 808-814.

Hertweck M. 2005. C. elgans gives the dirt on aging. Science of Aging Knowledge Environment 2005(41): pe31.

Hiona A and Leeuwenburgh C. 2008. The role of mitochondrial DNA mutations in aging and sarcopenia: Implications for the mitochondrial vicious cycle theory of aging. Experimental Gerontology 43: 24-33.

Houthoofd K and Vanfleteren JR. 2007. Public and private mechanisms of life extension in Caenorhabditis elegans. Molecular Genetics and Genomics 277: 601-617.

Howe DK and Denver DR. 2008. Muller's ratchet and compensatory mutation in Caenorhabditis briggsae mitochondrial genome evolution. BMC Evolutionary Biology 2008, 8: 62. 
Howe DK, Baer CF, and Denver DR. 2010. High rate of large deletions in Caenorhabditis briggsae mitochondrial genome mutation processes. Genome Biology and Evolution 1(2): 29-38.

Huang C, Xiong C, and Kornfeld K. 2004. Measurements of age-related changes of physiological processes that predict lifespan of Caenorhabditis elegans.

Proceedings of the National Academies of Science, USA 101(21): 8084-8089.

Inoue K, Nakada K, Ogura A, Isobe K, Goto Y, Nonaka I, and Hayashi JI. 2000. Generation of mice with mitochondrial dysfunction by introducing mouse mtDNA carrying a deletion into zygotes. Nature Genetics 26: 176-181.

Janssen RJRJ, Nijtmans LG, van den Heuvel LP, and Smeitink JAM. 2006. Mitochondrial complex I: Structure, function and pathology. Journal of Inherited Metabolic Disease 29(4): 499-515.

Kayser EB, Sedensky MM and Morgan PG. 2004. The effects of complex I function and oxidative damage on lifespan and anesthetic sensitivity in Caenorhabditis elegans. Mechanisms of Ageing and Development 125: 455-464.

Kimura K, Tanaka N, Nakamura N, Takano S, and Ohkuma S. 2007. Knockdown of mitochondrial heat shock protein 70 promotes progeria-like phenotypes in Caenorhabditis elegans. Journal of Biological Chemistry 282(8): 5910-5918.

Krishnan KJ, Reeve AK, Samuels DC, Chinnery PF, Blackwood JK, Taylor RW, Wanrooij S, Spelbrink JN, Lightowlers RN, and Turnbull DM. 2008. What causes mitochondrial DNA deletions in human cells? Nature Genetics 40(3): 275-279.

Lapointe J and Hekimi S. 2010 When a theory of aging ages badly. Cellular and Molecular Life Sciences 67: 1-8.

Lee SS, Lee RYN, Fraser AG, Kamath RS, Ahringer J, and Ruvkun G. 2003. A systematic RNAi screen identifies a critical role for mitochondria in C. elegans longevity. Nature Genetics 33: 40-48.

Lee HC and Wei YH. 2007. Oxidative stress, mitochondrial DNA mutations, and apoptosis in aging. Experimental Biology and Medicine, 227(9): 671-682.

Lemire B. 2005. Mitochondrial genetics, WormBook, ed. The C. elegans Research Community, WormBook, doi/10.1895/wormbook.1.25.1, http://www.wormbook.org.

Lemire BD, Behrendt M, DeCorby A, and Gaskova D. 2009. C. elegans longevity pathways converge to decrease mitochondrial membrane potential. Mechanisms of Ageing and Development 130: 461-465.

Liau WS, Gonzalez-Serricchio AS, Deshommes C, Chin K, and LaMunyon CW. 2007. A persistent mitochondrial deletion reduces fitness and sperm performance in heteroplasmic populations of C. elegans. BMC Genetics 8: 8.

Lightowlers RN, Chinnery PF, Turnbull DM, and Howell N. 1997. Mammalian mitochondrial genetics: heredity, heteroplasmy and disease. Trends in Genetics 13(11): 450-455. 
Linnane AW, Marzuki S, Ozawa T, and Tanaka M. 1989. Mitochondrial DNA mutations as an important contributor to ageing and degenerative diseases. The Lancet 333: 642-645.

Luo X, Pitkanen S, Kassovska-Bratinova S, Robinson BH, and Lehotay DC. 1997. Excessive formation of hydroxyl radicals and aldehydic lipid peroxidation products in cultured skin fibroblasts from patients with complex I deficiency. Journal of Clinical Investigation 99(12): 2877-2882.

Lunt DH and Hyman BC. 1997. Animal mitochondrial DNA recombination. Nature 387: 247-247.

Lynch M. 1991. The genetic interpretation of inbreeding depression and outbreeding depression. Evolution 45(3): 622-629.

Melov S, Lithgow GJ, Fischer DR, Tedesco PM, and Johnson TE. 1995. Increased frequency of deletions in the mitochondrial genomes with age of Caenorhabditis elegans. Nucleic Acids Research 23(8): 1419-1425.

Melov S, Hinerfeld D, Esposito L, and Wallace DC. 1997. Multi-organ characterization of mitochondrial genomic rearrangements in ad libitum and caloric restricted mice show striking somatic mitochondrial DNA rearrangements with age. Nucleic Acids Research 25(5): 974-982.

Miaw S, Beckman KB, and Muller FL, eds. 2008. Oxidative Stress in Aging: From Model Systems to Human Diseases. Humana Press, Totowa, NJ, USA.

Moraes CT. 2001. What regulates mitochondrial DNA copy number in animal cells? Trends in Genetics 17(4): 199-205.

Murphy MP. 2009. How mitochondria produce reactive oxygen species. Biochemical Journal 417:1-9.

Ozawa T. 1995. Mechanism of somatic mitochondrial DNA mutations associated with age and diseases. Biochemica et Biophysica Acta 1271: 177-189.

Phillips P, Salomon M, Custer A, Ostrow D, and Baer CR. 2009. Spontaneous mutational and standing genetic (co)variation at dinucleotide microsatellites in Caenorhabditis briggsae and Caenorhabditis elegans. Molecular Biology and Evolution 26: 659-669.

Pitkanen S and Robinson BH. 1996. Mitochondrial complex I deficiency leads to increased production of superoxide radicals and induction of superoxide dismutase. Journal of Clinical Invesitgation 98(2): 345-351.

R Development Core Team. 2009. R: A language and environment for statistical computing. R Foundation for Statistical Computing, Vienna, Austria. ISBN 3900051-07-0, URL http://www.R-project.org.

Rand DM, Haney RA, and Fry AJ. 2004. Cytonuclear coevolution: the genomics of cooperation. Trends in Ecology and Evolution 19: 645-653.

Rea SL. 2005. Metabolism in the Caenorhabditis elegans Mit mutants. Experimental Gerontology 40: 841-849. 
Rea SL and Johnson TE. 2003. A metabolic model for lifespan determination in Caenorhabditis elegans. Developmental Cell 5: 197-203.

Rea SL, Venture N, and Johnson TE. 2007. Relationship between mitochondrial electron transport chain dysfunction, development, and life extension in Caenorhabditis elegans. Public Library of Science 5(10): 2312-2329.

Robert KA, Brunet-Rossinni A, and Bronikowski AM. 2007. Testing the 'free radical theory of aging' hypothesis: physiological differences in long-lived and shortlived colubrid snakes. Aging Cell 6: 395-404.

Rossignol R, Faustin B, Rocher C, Malgat M, Mazat JP, and Letellier T. 2003. Mitochondrial threshold effects . Biochemical Journal 370: 751-762.

Schierup MH and Christiansen FB. 1996. Inbreeding depression and outbreeding depression in plants. Heredity 77:461-468.

Sedensky MM and Morgan PG. 2006a. Mitochondrial respiration and reactive oxygen species in C. elegans. Experimental Gerontology 41: 957-967.

Sedensky MM and Morgan PG. 2006b. Mitochondrial respiration and reactive oxygen species in mitochondrial aging mutants. Experimental Gerontology 41: 237-245.

Smeitink J, van dan Heuvel L, and DiMauro S. 2001. The genetics and pathology of oxidative phosphorylation. Nature Reviews Genetics 2: 342-352.

Solignac M, Genermont J, Monnerot M, and Mounolou JC. 1987. Drosophila mitochondrial genetics: evolution of heteroplasmy through germ line cell divisions. Genetics 117: 687-696.

Sutovsky P. 2003. Degradation of paternal mitochondrial after fertilization: implications for heteroplasmy, assisted reproductive technologies and mtDNA inheritance. Reproductive BioMedicine Online 8(1): 24-33.

Taylor RW and Turnbull DM. 2005. Mitochondrial DNA mutations in human disease. Nature Reviews Genetics 6: 389-402.

Tsang WY and Lemire BD. 2002. Stable heteroplasmy but differential inheritance of a large mitochondrial DNA deletion in nematodes. Biochemistry and Cell Biology 80: 645-654.

Tsang WY and Lemire BD. 2003. The role of mitochondria in the life of the nematode, Caenorhabditis elegans. Biochemica et Biophysica Acta 1638: 91-105.

Venables WN and Ripley BD. 2002. Modern Applied Statistics with S. Fourth Edition. Springer, New York. ISBN 0-387-95457-0.

Ventura N, Rea SL and Testi R. 2006. Long-lived C. elegans mitochondrial mutants as a model for human mitochondrial-associated diseases. Experimental Gerontology 41: 974-991.

Wallace DC. 2005. A mitochondrial paradigm of metabolic and degenerative diseases, aging and cancer: a dawn for evolutionary medicine. Annual Reviews of Genetics 39: 359-407. 
Willet CS and Burton RS. 2001. Viability of cytochrome $c$ genotypes depends on cytoplasmic backgrounds in Tigriopus californicus. Evolution 55: 1592-1599.

Willett CS and Burton RS. 2004. Evolution of interacting proteins in the mitochondrial electron transport system in a marine copepod. Molecular Biology and Evolution 21(3): 443-453.

Wolf JB. 2009. Cytonuclear interactions can favor the evolution of genomic imprinting. Evolution 63(5): 1364-1371.

Yoneda M, Chomyn A, Martinuzzi A, Hurko O, and Attardi G. 1992. Marked replicative advantage of human mtDNA carrying a point mutation that causes the MELAS encephalomyopathy. Proceedings of the National Academies of Science, USA 89: 11164-11168. 